\title{
Remittances and the Brain Drain: Evidence from Microdata for Sub-Saharan Africa
}

\author{
Julia Bredtmann, Fernanda Martínez Flores \& Sebastian Otten
}

To cite this article: Julia Bredtmann, Fernanda Martínez Flores \& Sebastian Otten (2019)

Remittances and the Brain Drain: Evidence from Microdata for Sub-Saharan Africa, The Journal of Development Studies, 55:7, 1455-1476, DOI: 10.1080/00220388.2018.1443208

To link to this article: https://doi.org/10.1080/00220388.2018.1443208
(C) 2018 The Author(s). Published by Informa UK Limited, trading as Taylor \& Francis Group.

\section{+ View supplementary material $[ح$}

\section{曲 Published online: 11 Mar 2018.}

Submit your article to this journal $\pi$

LII Article views: 1970

View Crossmark data $\nearrow$

的

Citing articles: 2 View citing articles $\longleftarrow$ 


\title{
Remittances and the Brain Drain: Evidence from Microdata for Sub-Saharan Africa
}

\author{
JULIA BREDTMANN $* * *, \dagger$, FERNANDA MARTÍNEZ FLORES $*$, \\ \& SEBASTIAN OTTEN $*, \dagger, \S$ \\ *RWI - Leibniz Institute for Economic Research, Essen, Germany, **IZA - Institute of Labor Studies, Bonn, Germany, \\ ${ }^{\dagger}$ Centre for Research and Analysis of Migration (CReAM), London, UK, ${ }^{*}$ Ruhr University Bochum, Bochum, Germany, \\ ${ }^{\S}$ University College London, London, UK
}

(Original version submitted April 2017; final version accepted January 2018)

\begin{abstract}
Based on unique microdata from five Sub-Saharan African countries that contain comprehensive information on both migrants and their households at the origin country, we investigate the effect of migrants' education on their remittance behaviour. Our results reveal that migrants' education has no impact on the likelihood of sending remittances, but a positive effect on the amount of money sent, conditional on remitting. The latter effect holds for internal migrants and migrants in non-OECD countries, while it vanishes for migrants in OECD destination countries once characteristics of the origin household are controlled for.
\end{abstract}

\section{Introduction}

The so-called 'brain drain', or the migration of high-skilled individuals to other regions where human capital is abundant, is a major concern for developing countries with a relatively small number of highly educated individuals, as it represents the loss of their most talented workers (see, for example, Beine, Docquier, \& Rapoport, 2008; Di Maria \& Lazarova, 2012; Djiofack, Djimeu, \& Boussichas, 2013; Haque \& Kim, 1995). ${ }^{1}$ In this context, Sub-Saharan Africa is of particular interest: not only is the share of high-skilled individuals in Sub-Saharan Africa among the lowest in the world (UNESCO, 2016), but the high-skilled emigration rate from these countries has also steadily increased since the mid-1990s (from $11 \%$ in 1995 to $16 \%$ in 2010 , see Figure 1), representing today the highest high-skilled emigration rate among all developing regions. This evolution is amplified by the high ratio of high-skilled to low-skilled emigrants (in 2010, the low-skilled emigration rate from Sub-Saharan Africa was 0.5\%), indicating that migrants from Sub-Saharan Africa are particularly positively selected in terms of their education. The brain drain thus represents a major policy challenge in these countries, as it involves the transfer of human capital, which is one of their scarcest resources and a key determinant to economic growth and poverty reduction. A prominent example is the medical brain drain (for example, the emigration of doctors and nurses), which is particularly harmful because the number of physicians per inhabitant is extremely low in Sub-Saharan Africa, leading to a severe shortage of health-care workers and a decline in the population's health status (Docquier \& Rapoport, 2012). ${ }^{2}$

Correspondence Address: Julia Bredtmann, RWI - Leibniz Institute for Economic Research, Hohenzollernstr. 1-3, 45128 Essen, Germany. Email: julia.bredtmann@rwi-essen.de

Supplementary Materials are available for this article which can be accessed via the online version of this journal available at https://doi.org/10.1080/00220388.2018.1443208

(C) 2018 The Author(s). Published by Informa UK Limited, trading as Taylor \& Francis Group.

This is an Open Access article distributed under the terms of the Creative Commons Attribution License (http://creativecom mons.org/licenses/by/4.0/), which permits unrestricted use, distribution, and reproduction in any medium, provided the original work is properly cited. 


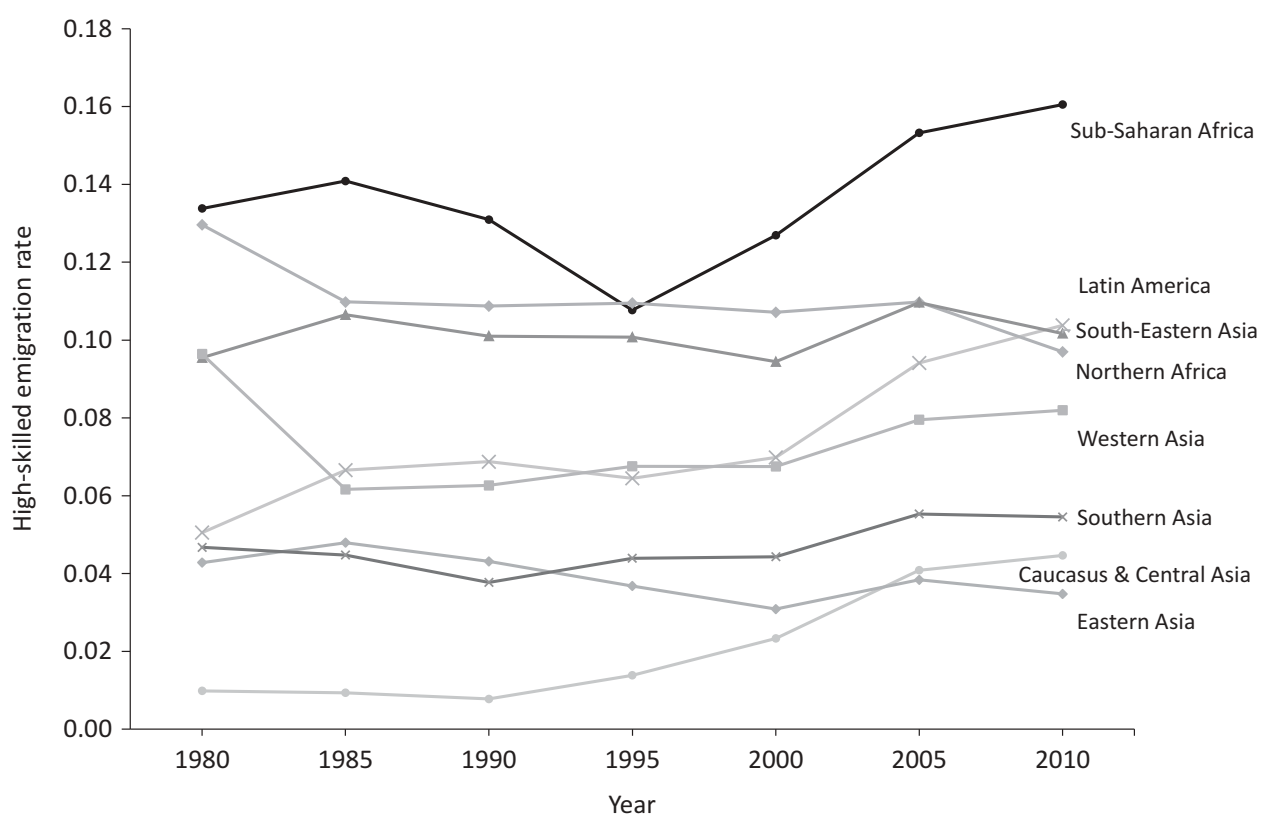

Figure 1. Evolution of high-skilled migration by region.

Source: Authors' analysis based on data from Brücker, Capuano, and Marfouk (2013).

The most evident way through which some of the negative externalities of the brain drain can be somewhat offset are remittances (Docquier \& Rapoport, 2012; Gibson \& McKenzie, 2012). Although numerous papers investigate the determinants of migrants' remittance patterns, ${ }^{3}$ there is still little consensus on whether and how the education of migrants affects the likelihood and the amount of remittances sent. Building on previous literature, we revisit this question and create a unique crosscountry dataset by combining household surveys from five Sub-Saharan African countries, which includes detailed information on both the migrants and the households at the origin country.

Theoretically, there are several reasons why high-skilled migrants may send more or less remittances than low-skilled migrants. On the one hand, high-skilled migrants may remit less because they often come from better-off families with lower income constraints. Besides, they may have a lower propensity to return to their origin countries, which decreases their incentives to invest in their home community. On the other hand, more educated migrants are subject to higher wages which, according to the altruism motive of remittances, would predict them to remit more. Also, they face lower transaction costs because they are more likely to have access to bank accounts and other financial services, and are less likely to be undocumented migrants. In addition, following the investment motive, remittances may serve as a way of repayment if family members at the origin country have funded the education of the migrant (Docquier \& Rapoport, 2012; Rapoport \& Docquier, 2006). It is, thus, not clear if highly educated migrants remit more or less than less skilled migrants.

Previous empirical literature on the effect of education on remittance patterns mainly relies on macrodata, investigating the relationship between high-skilled emigration rates and remittance inflows at the country level. Limiting their analysis to OECD destination countries, Faini (2007), Adams (2009), and Niimi, Özden, and Schiff (2010) conclude that the adverse impact of skilled migration cannot be mitigated through remittances because migrant remittances are lower in countries with a high share of high skilled emigrants. These studies, however, are subject to a potential endogeneity bias because they only observe the aggregate amount of remittances received by each country. However, countries differ in many other ways. For example, if poverty is a constraint to both migration and education, poor countries might send less migrants (and thus receive fewer remittances) and those migrants might also be less educated, creating a spurious relationship between a country's 
high-skilled emigration rate and the amount of remittances received. Docquier, Rapoport, and Salomone (2012) and Le Goff and Salomone (2016) addresses this issue by using panel data on bilateral remittances. Both studies find a positive relationship between the share of high-skilled migrants and the amount of remittances received. Docquier et al. (2012) further show that the destination country's immigration policy is a key determinant of the sign and magnitude of the relationship between high-skilled emigration rates and remittances.

As outlined by Bollard, McKenzie, Morten, and Rapoport (2011), the main disadvantage of analyses based on macrodata is that they are only able to identify whether countries that send a larger share of educated migrants receive larger or smaller remittance flows than countries sending a smaller share of high-skilled migrants. Microdata, on the other hand, allows a direct investigation of the relationship between migrants' education and their remittance behaviour. Furthermore, it has the advantage of capturing remittances sent through both formal and informal channels. Empirical evidence based on microdata, however, is still scarce and the results are inconclusive.

Dustmann and Mestres (2010) use data from the German Socio-Economic Panel and find a negative effect of years of schooling on remittances after controlling for return intentions and for whether family members (spouse and children) still live in the origin country. Bollard et al. (2011), on the other hand, using a pooled dataset of household surveys in 11 OECD destination countries, find a mixed relationship between migrants' education and remittances. While they do not find a significant effect of having a university degree on the likelihood of sending remittances, they find a strong positive relationship between education and the amount of money sent, conditional on sending remittances. Using the same database but focusing on African migrants, Bollard, McKenzie, and Morten (2010) come to a similar conclusion and further show that the relationship between education and the amount remitted is non-linear.

A common issue that previous microdata studies share is that they are based on survey data collected in migrants' destination countries, and thus not able to incorporate the economic situation and the characteristics of the household left behind in their analysis. ${ }^{4}$ However, the economic conditions in migrants' home region and the characteristics of the origin household, for example, the income level, are important determinants of the remittance behaviour of migrants. ${ }^{5}$

We overcome these issues by analysing the effect of migrants' education on their remitting behaviour using unique household survey data from five Sub-Saharan African sending countries, namely Burkina Faso, Kenya, Nigeria, Senegal, and Uganda. This database has two main advantages: First, it contains comprehensive information on both the migrant and the household left behind, enabling us to control for characteristics of both sides of the migrant-household relationship. Second, previous studies concerning remittances and high-skilled migration focus only on individuals who migrated to OECD countries, which represent a very selective group of migrants. ${ }^{6}$ Yet, the emigration of high skilled individuals is not only reflected in migration flows from low-income to high-income countries, but is also a regional and local concern. For instance, the movement of highly educated individuals within national borders, usually from rural to urban areas, can result in a persistent state of underdevelopment and impoverishment (George, Dei, \& Asgharzadeh, 2002). To address this, we use a sample of all individuals having migrated from five sending countries to different destinations, including OECD countries, non-OECD countries as well as internal migrants. Hence, we are able to provide important insights into the remitting behaviour of the largest, but so far neglected groups of migrants - internal migrants and migrants in non-OECD destination countries. ${ }^{7}$

Overall, we do not find evidence that education is a determinant of the likelihood of sending remittances. It is, however, an important factor to determine the amount of remittances sent. Conditional on sending remittances, migrants with a university degree send a significantly higher amount than migrants with lower levels of education. These results are robust to a number of sensitivity analyses. This suggests that some of the negative externalities of the brain drain on the source countries can be counterbalanced by migrants' remittances. These effects hold for the subsamples of internal migrants and migrants in non-OECD countries. For migrants in OECD destination countries, however, the positive effect of education on the amount of remittances sent vanishes once characteristics of the household at the origin country are controlled for. This result highlights the 
importance of controlling for characteristics of both sides of the migrant-household relationship when analysing migrants' remittance behaviour.

The structure of the paper is as follows. The following section outlines the empirical methodology. Section 3 describes the data and presents some descriptive statistics. In Section 4, we discuss our estimation results and Section 5 concludes.

\section{Empirical model}

To identify the effect of migrants' education on their remittance behaviour, we estimate a model in which migrants' remittances are a function of a set of independent variables containing information on the migrants and their households left behind. In our baseline specification, we estimate the following model:

$$
R_{i j}=\alpha+\beta^{\prime} E_{i}+\theta^{\prime} \boldsymbol{X}_{\boldsymbol{i}}+\gamma^{\prime} \boldsymbol{H}_{\boldsymbol{j}}+\delta_{d}+\eta_{r}+\epsilon_{i j}
$$

where $R_{i j}$ measures remittances sent by migrant $i$ to household $j . E_{i}$ is a dummy variable indicating if the individual has a university degree or not, $\boldsymbol{X}_{\boldsymbol{i}}$ represents a vector of individual demographic characteristics, $\boldsymbol{H}_{\boldsymbol{j}}$ is a vector of characteristics of the household at the origin country, and $\epsilon_{i j}$ represents the error term. Our key coefficient of interest is $\beta$, the estimated impact of having a university degree on migrants' remittance behaviour.

To account for observed and unobserved heterogeneity at the destination (for example, labour market characteristics) and the origin level (for example, educational quality), we further add destination-country fixed effects, $\delta_{d}$, and origin-region fixed effects at the district level, $\eta_{r}$, to the estimation model. Destination and origin-region fixed effects are also important to control for the selection of specific types of individuals into migration and their sorting into specific destination countries (Licuanan, Mahmoud, \& Steinmayr, 2015). For instance, migrants who have a high motivation to remit may choose to locate in countries with high wages. These countries may also primarily demand high-skilled migrants, because of their high labour market standards. As a result, the relationship between migrants' education and the level of remittances sent may be biased, because it merely reflects the type of migrants who choose to live in these countries. On the origin-region side, it is likely that regions that regularly face natural disasters, such as droughts and floods, receive more remittances. At the same time, it is likely that the educational infrastructure in these regions is comparatively underdeveloped and that migrants are mainly low skilled. If the origin region's exposure to natural disasters and other local characteristics are not controlled for, the estimated effect of migrants' education on their remittance behaviour is therefore likely to be biased.

In the above specification, we control for observed characteristics of the migrant and the household at the origin country. Of course, there might also exist some unobserved factors at both levels that we cannot account for. We might not be able to perfectly capture the local conditions of the area the origin household is located in, which might be correlated with both migrants' remittances and their level of education. Also, unobserved heterogeneity at the individual level, for example, due to ability, might still affect our estimates. Hence, while we are able to largely reduce the problem of unobserved heterogeneity across migrants' origin households that is present in other studies, we do not claim to estimate a true causal effect of migrants' education on their remittance behaviour.

Similar to Bollard et al. (2011), we use three alternative measures of remittances. First, in order to capture the overall effect of migrants' education on remittances, we use the reported total amount of remittances to capture both the extensive and intensive margin. Second, we create a binary variable that indicates whether the migrant remits or not (extensive margin). Third, we use the logarithm of the amount of remittances conditional on remitting (intensive margin). For all three outcomes, the model is estimated using OLS. ${ }^{8}$ To account for within-household correlation, the standard errors are clustered at the origin-household level. 


\section{Data}

\subsection{The migration and remittances household surveys}

The data used in our empirical analysis comes from the Migration and Remittances Households Surveys conducted in 2009-2010 by the World Bank in six Sub-Saharan African countries: Burkina Faso, Kenya, Nigeria, Senegal, Uganda, and South Africa (Plaza, Navarrete, \& Ratha, 2011). The single household surveys are part of the Africa Migration Project and are standardised across countries, which allows us to combine them to a unique cross-country dataset. ${ }^{9}$ An exception is the survey for South Africa, which differs because South Africa represents a migrant-receiving country rather than a migrant-sending country, and is therefore excluded from our analysis.

In each country, about 2000 households were interviewed and comprehensive information on the household at the origin country as well as on the characteristics of all household members was collected - of those still living in the household and of former household members who migrated. As highlighted by Osili (2007) and De Brauw, Mueller, and Woldehanna (2013), having data on both the sending and the receiving household is crucial for understanding migrants' remittance behaviour. While previous studies on the brain drain and migrants' remittance behaviour observe only one side of the migrant-origin family transaction, we are the first to be able to consider comprehensive information on both sending and receiving households. In particular, the data contains information on the level of remittances sent by each former household member to the origin household. In contrast to macro databases on bilateral remittances, which only capture remittances sent through formal channels, this includes remittances sent through both formal and informal channels and thus represents a more reliable measure of migrants' remittances (Clemens \& McKenzie, 2018).

In addition, a main feature of the data is that it not only reports information on migrants who live abroad, but also on internal migrants, who mainly moved from rural to urban areas. Hence, in contrast to previous studies, which only consider migrants to OECD countries, we observe a sample of all individuals who have migrated from five sending countries to different destinations, including OECD countries, non-OECD countries as well as internal migrants. Having data from migrants' sending countries further enables us to consider undocumented migrants, which are usually not included in surveys collected in migrants' destination countries.

Household surveys collected in migrants' sending countries have the disadvantage, though, that they are likely to miss migrants who emigrated with their entire household. However, as Bollard et al. (2011) show, household composition in the destination country is about the same for high- and low-skilled migrants, which makes us confident that this sort of selectivity will hardly affect our estimation results.

For the purpose of our identification strategy, we restrict the sample to households that report to have at least one former household member that migrated before the interview was conducted. Moreover, we restrict our sample to migrants aged 24 and older, the age at which most individuals should have finished a (undergraduate) university degree. Considering both internal and international migrants, the overall sample consists of 7536 migrants from 4460 origin households.

\subsection{Variables}

With respect to the dependent variable, migrants' remittances, the households report the amount of remittances received from each migrant in the last 12 months before the interview was conducted. In order to make remittance and income levels comparable across countries, we convert all financial values to US dollars using the average exchange rate for 2009 for each local currency. ${ }^{10}$

Regarding our variable of interest, we create a binary variable indicating whether the individual has a university degree or completed postgraduate studies. All individuals with lower education levels are included in the reference group. With respect to migrants' demographic characteristics, we control for gender, age, marital status, and years spent at the destination country. To allow for non-linear effects, the latter two variables are further included as squared terms. In addition, we include a binary variable indicating if the individual migrated internally. We also control for the migration reason by indicating 
if the decision was made to search for a job, to pursue education, to reunite with family members, or for other reasons (for example, related to conflict or weather conditions).

Unfortunately, we do not have information on the migrant's income level at the destination country. As argued, amongst others, by Bollard et al. (2011), income should be one of the main channels through which education affects remittances. We aim to address this problem by controlling for factors that are highly correlated with migrants' income and that can thus serve as a proxy for their unobserved earnings potential. Specifically, we control for the current labour force status of the migrant, distinguishing between full-time employment, part-time employment, self-employment, and not participating in the labour force. In addition, we have information on migrants' occupation at the destination country. However, as this information is available for a large fraction, but not for all migrants in our sample, we refrain from using it in our baseline regressions, but rather conduct a robustness check in which we control for migrants' occupation level (see Section 4.2). We argue that once migrants' education, their labour force status, their occupation and their destination country (see below) are accounted for, migrants' income should have a minor impact on their remittance behaviour. Nevertheless, when interpreting the results, it should be kept in mind that part of the effect of migrants' education on remittances might represent an indirect effect that operates through migrants' income.

We also control for the relationship of the migrant to the current household head in the origin country. We distinguish between spouses, children, and other relatives of the household head, whom we expect to have different motives to remit. Finally, we add indicator variables for different groups of destination countries, which we categorise in four groups according to their income level: high, uppermiddle, lower-middle and low-income (see World Bank, 2015). Alternatively, we include destinationcountry fixed effects to control for unobserved characteristics of the destination country.

We further control for a comprehensive set of characteristics of the households at the origin country. We proxy the non-remittance income by total household expenditures minus remittance income. ${ }^{11}$ As the time base for different expenditures varies over the categories, we aggregate weekly and monthly values to yearly values, to obtain a measure of the household's total expenditures. We also include additional controls that are related to the socio-economic status of the family or its wealth, that is, indicators for house ownership, agricultural land ownership, and access to electricity. Furthermore, we account for the demographic composition of the household by including household size, the number of migrants, a dummy variable indicating if the household head is male, and the share of dependents (children, unemployed, and elderly people). We build the latter variable by aggregating the number of household members who are not part of the labour force and dividing it by the total household size. ${ }^{12}$

\section{Results}

\subsection{Baseline results}

The results of estimating Equation (1) are presented in Tables 1-3. We start our analysis by estimating the effect of education on the total amount of remittances sent using a sample of all migrants, that is, unconditional on whether the migrants send remittances or not. Column $I$ in Table 1 shows a parsimonious specification that controls for migrant characteristics, origin-country fixed effects, and the income group of the destination country. The results reveal that migrants with a university degree remit, on average, about 304 USD more than less educated migrants. When including destinationcountry fixed effects to control for destination-specific factors, such as differences in wages or employment probabilities, the coefficient slightly decreases in magnitude but remains similar in terms of significance (column II). In subsequent columns, we stepwise add the households' level of non-remittance income and further important household characteristics to the model. When controlling for household income (column III), which itself is negatively correlated with migrants' remittances, the estimated effect of having a university degree increases to 362 USD. This reveals that indeed, migrants from high-income households do remit less and are more highly educated, which creates a spurious relationship between migrants' education and their remittance flows. When adding further originhousehold characteristics (column IV) as well as district fixed effects to capture the large regional 
Table 1. Determinants of total remittances

\begin{tabular}{|c|c|c|c|c|c|c|}
\hline & I & II & III & IV & $\mathrm{V}$ & VI \\
\hline \multicolumn{7}{|l|}{ Migrant characteristics } \\
\hline University degree & $\begin{array}{l}304.347 * * \\
(121.782)\end{array}$ & $\begin{array}{l}264.887 * * \\
(115.750)\end{array}$ & $\begin{array}{l}362.047^{* * *} \\
(111.322)\end{array}$ & $\begin{array}{l}310.308^{* * *} \\
(111.833)\end{array}$ & $\begin{array}{l}266.056^{* *} \\
(116.173)\end{array}$ & $\begin{array}{l}324.550 * * * \\
(114.713)\end{array}$ \\
\hline Male & $\begin{array}{l}113.812 * * \\
(53.747)\end{array}$ & $\begin{array}{l}108.770^{* *} \\
(54.254)\end{array}$ & $\begin{array}{c}76.101 \\
(53.706)\end{array}$ & $\begin{array}{c}53.636 \\
(53.065)\end{array}$ & $\begin{array}{c}57.101 \\
(53.169)\end{array}$ & $\begin{array}{c}78.553 \\
(52.268)\end{array}$ \\
\hline Age/100 & $\begin{array}{c}1767.159 \\
(1264.618)\end{array}$ & $\begin{array}{c}1707.245 \\
(1221.513)\end{array}$ & $\begin{array}{c}1201.858 \\
(1165.878)\end{array}$ & $\begin{array}{c}1891.96 \\
(1195.994)\end{array}$ & $\begin{array}{c}1845.445 \\
(1169.339)\end{array}$ & $\begin{array}{l}2747.436^{* *} \\
(1177.177)\end{array}$ \\
\hline $\operatorname{Age}^{2} / 100$ & $\begin{array}{c}-11.562 \\
(15.612)\end{array}$ & $\begin{array}{c}-11.498 \\
(15.057)\end{array}$ & $\begin{array}{c}-5.874 \\
(14.511)\end{array}$ & $\begin{array}{c}-12.640 \\
(14.694)\end{array}$ & $\begin{array}{c}-11.518 \\
(14.345)\end{array}$ & $\begin{array}{c}-21.777 \\
(14.399)\end{array}$ \\
\hline Married & $\begin{array}{l}-35.108 \\
(66.575)\end{array}$ & $\begin{array}{c}-41.209 \\
(67.092)\end{array}$ & $\begin{array}{r}-87.859 \\
(66.142)\end{array}$ & $\begin{array}{r}-94.680 \\
(65.430)\end{array}$ & $\begin{array}{r}-87.677 \\
(65.218)\end{array}$ & $\begin{array}{c}-53.971 \\
(64.130)\end{array}$ \\
\hline Years since emigration/100 & $\begin{array}{r}2864.915^{\dagger} \\
(696.883)\end{array}$ & $\begin{array}{r}2928.082^{\dagger} \\
(672.943)\end{array}$ & $\begin{array}{r}2610.332^{\dagger} \\
(616.723)\end{array}$ & $\begin{array}{r}2868.509^{\dagger} \\
(651.877)\end{array}$ & $\begin{array}{r}2525.085^{\dagger} \\
(626.797)\end{array}$ & $\begin{array}{r}2766.126^{\dagger} \\
(668.647)\end{array}$ \\
\hline Years since emigration ${ }^{2} / 100$ & $\begin{array}{l}-62.447 * * * \\
(20.708)\end{array}$ & $\begin{array}{l}-61.392 * * * \\
(19.630)\end{array}$ & $\begin{array}{l}-52.301 * * * \\
(17.287)\end{array}$ & $\begin{array}{l}-56.366^{* * *} \\
(18.343)\end{array}$ & $\begin{array}{l}-48.229^{* * *} \\
(16.677)\end{array}$ & $\begin{array}{l}-52.743 * * * \\
(18.567)\end{array}$ \\
\hline Internal migrant & $\begin{array}{r}-220.636^{\dagger} \\
(56.334)\end{array}$ & $\begin{array}{l}-305.913 \\
(221.937)\end{array}$ & $\begin{array}{l}-277.224 \\
(227.159)\end{array}$ & $\begin{array}{l}-225.955 \\
(231.447)\end{array}$ & $\begin{array}{c}-217.639 \\
(241.298)\end{array}$ & $\begin{array}{l}-205.398 \\
(242.110)\end{array}$ \\
\hline \multicolumn{7}{|c|}{ Migration reason (Ref: Work related) } \\
\hline Education & $\begin{array}{r}-122.057 \\
(89.802)\end{array}$ & $\begin{array}{r}-159.077 \\
(96.764)\end{array}$ & $\begin{array}{r}-128.635 \\
(87.971)\end{array}$ & $\begin{array}{r}-115.485 \\
(86.455)\end{array}$ & $\begin{array}{r}-149.851^{*} \\
(87.525)\end{array}$ & $\begin{array}{c}-261.995^{* * *} \\
(88.064)\end{array}$ \\
\hline Family & $\begin{array}{l}-9.841 \\
(81.900)\end{array}$ & $\begin{array}{l}-6.367 \\
(81.697)\end{array}$ & $\begin{array}{c}17.562 \\
(80.200)\end{array}$ & $\begin{array}{c}18.319 \\
(78.479)\end{array}$ & $\begin{array}{c}-49.369 \\
(80.231)\end{array}$ & $\begin{array}{c}-159.948^{* *} \\
(74.755)\end{array}$ \\
\hline Other & $\begin{array}{c}-162.757 \\
(106.982)\end{array}$ & $\begin{array}{c}-188.992 * \\
(101.406)\end{array}$ & $\begin{array}{c}-168.113 \\
(102.974)\end{array}$ & $\begin{array}{c}-164.569 \\
(104.706)\end{array}$ & $\begin{array}{c}-193.083^{*} \\
(104.016)\end{array}$ & $\begin{array}{l}-261.176^{* *} \\
(103.316)\end{array}$ \\
\hline \multicolumn{7}{|c|}{ Labour force status (Ref: Full time employed) } \\
\hline Part time employed & $\begin{array}{r}-396.725^{\dagger} \\
(90.701)\end{array}$ & $\begin{array}{r}-421.848^{\dagger} \\
(94.691)\end{array}$ & $\begin{array}{r}-434.447^{\dagger} \\
(82.452)\end{array}$ & $\begin{array}{r}-424.955^{\dagger} \\
(80.070)\end{array}$ & $\begin{array}{r}-402.683^{\dagger} \\
(77.712)\end{array}$ & - \\
\hline Self employed & $\begin{array}{r}-268.703^{\dagger} \\
(53.473)\end{array}$ & $\begin{array}{r}-274.616^{\dagger} \\
(53.278)\end{array}$ & $\begin{array}{r}-277.084^{\dagger} \\
(50.250)\end{array}$ & $\begin{array}{r}-254.268^{\dagger} \\
(50.086)\end{array}$ & $\begin{array}{r}-316.401^{\dagger} \\
(52.326)\end{array}$ & - \\
\hline Not in labour force & $\begin{array}{r}-503.773^{\dagger} \\
(69.164)\end{array}$ & $\begin{array}{r}-501.718^{\dagger} \\
(69.008)\end{array}$ & $\begin{array}{r}-491.095^{\dagger} \\
(69.913)\end{array}$ & $\begin{array}{r}-480.211^{\dagger} \\
(65.986)\end{array}$ & $\begin{array}{r}-445.952^{\dagger} \\
(67.582)\end{array}$ & - \\
\hline \multicolumn{7}{|c|}{ Relationship to head (Ref: Child) } \\
\hline Partner & $\begin{array}{c}872.764^{\dagger} \\
(133.360)\end{array}$ & $\begin{array}{r}856.252^{\dagger} \\
(134.678)\end{array}$ & $\begin{array}{c}781.819^{\dagger} \\
(133.108)\end{array}$ & $\begin{array}{r}775.176^{\dagger} \\
(129.615)\end{array}$ & $\begin{array}{r}727.787^{\dagger} \\
(128.382)\end{array}$ & $\begin{array}{c}737.424^{\dagger} \\
(128.745)\end{array}$ \\
\hline Sibling & $\begin{array}{r}-178.376^{\dagger} \\
(47.080)\end{array}$ & $\begin{array}{r}-170.931^{\dagger} \\
(48.252)\end{array}$ & $\begin{array}{r}-157.621^{\dagger} \\
(46.096)\end{array}$ & $\begin{array}{r}-216.933^{\dagger} \\
(47.498)\end{array}$ & $\begin{array}{r}-201.391^{\dagger} \\
(50.364)\end{array}$ & $\begin{array}{r}-199.976^{\dagger} \\
(50.853)\end{array}$ \\
\hline Other relative & $\begin{array}{r}-110.421 * \\
(60.091)\end{array}$ & $\begin{array}{r}-100.386^{*} \\
(59.860)\end{array}$ & $\begin{array}{c}-150.760 * * \\
(58.940)\end{array}$ & $\begin{array}{c}-184.884 * * * \\
(59.081)\end{array}$ & $\begin{array}{c}-151.461 * * \\
(61.988)\end{array}$ & $\begin{array}{c}-158.482^{* *} \\
(62.195)\end{array}$ \\
\hline \multicolumn{7}{|c|}{ Destination (Ref: Low income country) } \\
\hline High income & $\begin{array}{r}842.737^{\dagger} \\
(129.577)\end{array}$ & - & - & - & - & - \\
\hline Upper-middle income & $\begin{array}{l}521.760^{* * *} \\
(168.709)\end{array}$ & - & - & - & - & - \\
\hline Lower-middle income & $\begin{array}{c}-130.289^{* *} \\
(56.866)\end{array}$ & - & - & - & - & - \\
\hline \multicolumn{7}{|l|}{ Origin $\mathrm{HH}$ characteristics } \\
\hline $\ln ($ non-remittance income) & - & - & $\begin{array}{r}-179.887^{\dagger} \\
(25.050)\end{array}$ & $\begin{array}{r}-198.641^{\dagger} \\
(25.939)\end{array}$ & $\begin{array}{r}-213.611^{\dagger} \\
(27.202)\end{array}$ & $\begin{array}{r}-214.228^{\dagger} \\
(27.410)\end{array}$ \\
\hline Urban & - & - & - & $\begin{array}{c}98.089^{*} \\
(55.497)\end{array}$ & $\begin{array}{l}112.143^{*} \\
(66.359)\end{array}$ & $\begin{array}{l}134.240^{* *} \\
(66.950)\end{array}$ \\
\hline Number of migrants & - & - & - & $\begin{array}{r}-41.674^{\dagger} \\
(11.798)\end{array}$ & $\begin{array}{l}-46.096^{* * *} \\
(14.440)\end{array}$ & $\begin{array}{l}-45.619 * * * \\
(15.197)\end{array}$ \\
\hline $\mathrm{HH}$ head is male & - & - & - & $\begin{array}{c}50.059 \\
(59.113)\end{array}$ & $\begin{array}{c}72.167 \\
(58.142)\end{array}$ & $\begin{array}{c}79.811 \\
(58.867)\end{array}$ \\
\hline House is owned & - & - & - & $\begin{array}{c}21.714 \\
(76.208)\end{array}$ & $\begin{array}{l}137.272 \\
(89.360)\end{array}$ & $\begin{array}{l}159.036^{*} \\
(92.250)\end{array}$ \\
\hline Land is owned & - & - & - & $\begin{array}{c}-93.248 \\
(63.057)\end{array}$ & $\begin{array}{c}52.681 \\
(62.715)\end{array}$ & $\begin{array}{c}69.417 \\
(64.497)\end{array}$ \\
\hline Electricity & - & - & - & $\begin{array}{l}185.409 * * * \\
(59.244)\end{array}$ & $\begin{array}{l}145.870^{*} \\
(77.661)\end{array}$ & $\begin{array}{l}157.871 * * \\
(77.218)\end{array}$ \\
\hline HH size & - & - & - & $\begin{array}{r}31.046^{\dagger} \\
(5.081)\end{array}$ & $\begin{array}{l}32.032^{\dagger} \\
(5.629)\end{array}$ & $\begin{array}{r}31.584^{\dagger} \\
(5.681)\end{array}$ \\
\hline Share of dependents & - & - & - & $\begin{array}{c}-79.513 \\
(98.802)\end{array}$ & $\begin{array}{c}6.716 \\
(98.892)\end{array}$ & $\begin{array}{c}16.752 \\
(99.257)\end{array}$ \\
\hline
\end{tabular}


Table 1. (Continued)

\begin{tabular}{lcccccc}
\hline & I & II & III & IV & V & VI \\
\hline Constant & -159.428 & -284.764 & $1178.916 \dagger$ & $989.638 \dagger$ & $1343.401 \dagger$ & $772.776^{* *}$ \\
& $(255.122)$ & $(243.714)$ & $(288.767)$ & $(277.943)$ & $(376.194)$ & $(370.191)$ \\
Destination FE & no & yes & yes & yes & yes & yes \\
Origin FE & yes & yes & yes & yes & no & No \\
District FE & no & no & no & no & yes & yes \\
Observations & 7,536 & 7,536 & 7,536 & 7,536 & 7,536 & 7,536 \\
Adj- $\mathrm{R}^{2}$ & 0.138 & 0.145 & 0.187 & 0.196 & 0.217 & 0.211 \\
\hline
\end{tabular}

Notes: Results are obtained from OLS regressions. Standard errors in parentheses (clustered at the origin-household level). ${ }^{\dagger} \mathrm{p}<0.001 ;{ }^{* * *} \mathrm{p}<0.01 ;{ }^{* *} \mathrm{p}<0.05 ;{ }^{*} \mathrm{p}<0.1$.

heterogeneity within the origin countries (column V), the estimated education effect decreases to 310 USD and 266 USD, respectively. This suggests that both the economic situation of the household left behind and regional economic conditions at the origin country matter for migrants' remittance behaviour and are correlated with their degree of education obtained. Lastly, to test whether the effect of education operates through other channels such as income, we exclude the labour force status of the migrant from the estimation model (column VI). Indeed, the results show a strong increase in the estimated education effect, which implies that a large part of the impact of education on remittances works through the labour market success of the migrant in the destination country. In general, our results reveal that migrants with a university degree remit around 265-362 USD more per year than non-university educated migrants. Given that migrants remit 547 USD on average (see Table A1 in the Appendix), the difference in the amount of remittances sent by high- and low-skilled migrants is sizeable.

We next split the effect on the total level of remittances into two separate effects: the effect on the extensive margin and the effect on the intensive margin. The results for the extensive margin, migrants' probability to send remittances, are reported in Table 2. The estimated coefficients for having a university degree are negative and not statistically significant for all specifications, which reveals that the level of education is not a determinant of the decision to send remittances. Only when excluding the labour force status of the migrant from the estimation (column VI), the education effect turns positive, but is still not statistically significant. Hence, we do not find evidence that migrants' level of education is a determinant of their decision to remit.

The results for the intensive margin, the amount sent conditional on sending remittances, are reported in Table 3. For all specifications, the estimated effect of having a university degree is positive and highly significant. This reveals that, conditional on sending remittances, high-skilled migrants send higher amounts of money to their households left behind as compared to their lower skilled counterparts. In our basic specification (column I), the estimated education effect is about 0.51 , suggesting that migrants with a university degree remit about 51 per cent more than migrants without a university degree. This effect, however, largely decreases once origin-household characteristics (columns III and IV) are controlled for. In our preferred specification (column V), which further includes district fixed effects, migrants with a university degree remit around 31 per cent more than migrants without a university degree. Given that, conditional on remitting, migrants remit on average 991 USD (Table A1), high-skilled migrants send on average around 307 USD more than low-skilled migrants.

Overall, our results reveal that high-skilled migrants do send larger amounts of remittances than low-skilled migrants and thus contradict the findings of most of the previous literature based on macrodata (for example, Adams, 2009; Faini, 2007; Niimi et al., 2010). They are, though, in line with Bollard et al. (2011), who also find no robust effect of migrants' education on remittances at the extensive margin, but a positive effect at the intensive margin. However, our findings further reveal that part of the positive association between migrants' education and the amount of money sent is 
Table 2. Determinants of the probability of sending remittances

\begin{tabular}{|c|c|c|c|c|c|c|}
\hline & I & II & III & IV & $\mathrm{V}$ & VI \\
\hline \multicolumn{7}{|l|}{ Migrant characteristics } \\
\hline University degree & $\begin{array}{c}-0.030 \\
(0.023)\end{array}$ & $\begin{array}{c}-0.030 \\
(0.023)\end{array}$ & $\begin{array}{c}-0.016 \\
(0.022)\end{array}$ & $\begin{array}{c}-0.001 \\
(0.022)\end{array}$ & $\begin{array}{c}-0.006 \\
(0.021)\end{array}$ & $\begin{array}{c}0.022 \\
(0.022)\end{array}$ \\
\hline Male & $\begin{array}{l}0.039 * * * \\
(0.015)\end{array}$ & $\begin{array}{l}0.038^{* *} \\
(0.015)\end{array}$ & $\begin{array}{l}0.034 * * \\
(0.015)\end{array}$ & $\begin{array}{c}0.025^{*} \\
(0.015)\end{array}$ & $\begin{array}{c}0.019 \\
(0.014)\end{array}$ & $\begin{array}{r}0.053^{\dagger} \\
(0.015)\end{array}$ \\
\hline Age/100 & $\begin{array}{l}0.789 * * \\
(0.361)\end{array}$ & $\begin{array}{l}0.761^{* *} \\
(0.359)\end{array}$ & $\begin{array}{c}0.687^{*} \\
(0.355)\end{array}$ & $\begin{array}{c}0.602 * \\
(0.355)\end{array}$ & $\begin{array}{l}0.768^{* *} \\
(0.356)\end{array}$ & $\begin{array}{c}1.558^{\dagger} \\
(0.391)\end{array}$ \\
\hline $\mathrm{Age}^{2} / 100$ & $\begin{array}{c}-0.008^{*} \\
(0.004)\end{array}$ & $\begin{array}{c}-0.007^{*} \\
(0.004)\end{array}$ & $\begin{array}{c}-0.007 \\
(0.004)\end{array}$ & $\begin{array}{c}-0.006 \\
(0.004)\end{array}$ & $\begin{array}{c}-0.007^{*} \\
(0.004)\end{array}$ & $\begin{array}{r}-0.016^{\dagger} \\
(0.005)\end{array}$ \\
\hline Married & $\begin{array}{r}0.098^{\dagger} \\
(0.015)\end{array}$ & $\begin{array}{r}0.098^{\dagger} \\
(0.015)\end{array}$ & $\begin{array}{c}0.091^{\dagger} \\
(0.014)\end{array}$ & $\begin{array}{c}0.088^{\dagger} \\
(0.014)\end{array}$ & $\begin{array}{r}0.078^{\dagger} \\
(0.014)\end{array}$ & $\begin{array}{r}0.107^{\dagger} \\
(0.015)\end{array}$ \\
\hline Years since emigration/100 & $\begin{array}{l}0.442 * \\
(0.246)\end{array}$ & $\begin{array}{c}0.457^{*} \\
(0.247)\end{array}$ & $\begin{array}{c}0.410^{*} \\
(0.237)\end{array}$ & $\begin{array}{c}0.420^{*} \\
(0.245)\end{array}$ & $\begin{array}{l}0.437^{*} \\
(0.236)\end{array}$ & $\begin{array}{l}0.660^{* *} \\
(0.273)\end{array}$ \\
\hline Years since emigration ${ }^{2} / 100$ & $\begin{array}{c}-0.013^{*} \\
(0.007)\end{array}$ & $\begin{array}{c}-0.013^{*} \\
(0.008)\end{array}$ & $\begin{array}{c}-0.012 \\
(0.007)\end{array}$ & $\begin{array}{c}-0.012 \\
(0.007)\end{array}$ & $\begin{array}{c}-0.012^{*} \\
(0.007)\end{array}$ & $\begin{array}{c}-0.017^{*} \\
(0.009)\end{array}$ \\
\hline Internal migrant & $\begin{array}{c}0.003 \\
(0.018)\end{array}$ & $\begin{array}{c}-0.011 \\
(0.052)\end{array}$ & $\begin{array}{c}-0.007 \\
(0.051)\end{array}$ & $\begin{array}{c}-0.006 \\
(0.051)\end{array}$ & $\begin{array}{c}0.023 \\
(0.053)\end{array}$ & $\begin{array}{c}0.035 \\
(0.054)\end{array}$ \\
\hline \multicolumn{7}{|c|}{ Migration reason (Ref: Work related) } \\
\hline Education & $\begin{array}{r}-0.106^{\dagger} \\
(0.022)\end{array}$ & $\begin{array}{r}-0.114^{\dagger} \\
(0.022)\end{array}$ & $\begin{array}{r}-0.109^{\dagger} \\
(0.021)\end{array}$ & $\begin{array}{r}-0.102^{\dagger} \\
(0.021)\end{array}$ & $\begin{array}{r}-0.088^{\dagger} \\
(0.021)\end{array}$ & $\begin{array}{r}-0.216^{\dagger} \\
(0.021)\end{array}$ \\
\hline Family & $\begin{array}{r}-0.115^{\dagger} \\
(0.022)\end{array}$ & $\begin{array}{r}-0.118^{\dagger} \\
(0.022)\end{array}$ & $\begin{array}{r}-0.114^{\dagger} \\
(0.022)\end{array}$ & $\begin{array}{c}-0.102^{\dagger} \\
(0.021)\end{array}$ & $\begin{array}{c}-0.088^{\dagger} \\
(0.021)\end{array}$ & $\begin{array}{r}-0.194^{\dagger} \\
(0.021)\end{array}$ \\
\hline Other & $\begin{array}{c}-0.216^{\dagger} \\
(0.031)\end{array}$ & $\begin{array}{c}-0.212^{\dagger} \\
(0.031)\end{array}$ & $\begin{array}{c}-0.209^{\dagger} \\
(0.031)\end{array}$ & $\begin{array}{c}-0.197^{\dagger} \\
(0.030)\end{array}$ & $\begin{array}{r}-0.198^{\dagger} \\
(0.032)\end{array}$ & $\begin{array}{c}-0.257^{\dagger} \\
(0.032)\end{array}$ \\
\hline \multicolumn{7}{|c|}{ Labour force status (Ref: Full time employed) } \\
\hline Part time employed & $\begin{array}{c}-0.072 * * \\
(0.028)\end{array}$ & $\begin{array}{c}-0.069 * * \\
(0.028)\end{array}$ & $\begin{array}{l}-0.071 * * * \\
(0.027)\end{array}$ & $\begin{array}{l}-0.077 * * * \\
(0.027)\end{array}$ & $\begin{array}{c}-0.091^{\dagger} \\
(0.027)\end{array}$ & - \\
\hline Self employed & $\begin{array}{c}-0.104^{\dagger} \\
(0.017)\end{array}$ & $\begin{array}{c}-0.104^{\dagger} \\
(0.017)\end{array}$ & $\begin{array}{r}-0.105^{\dagger} \\
(0.017)\end{array}$ & $\begin{array}{c}-0.104^{\dagger} \\
(0.016)\end{array}$ & $\begin{array}{r}-0.095^{\dagger} \\
(0.016)\end{array}$ & - \\
\hline Not in labour force & $\begin{array}{r}-0.393^{\dagger} \\
(0.020)\end{array}$ & $\begin{array}{c}-0.391^{\dagger} \\
(0.021)\end{array}$ & $\begin{array}{r}-0.389^{\dagger} \\
(0.019)\end{array}$ & $\begin{array}{c}-0.388^{\dagger} \\
(0.020)\end{array}$ & $\begin{array}{c}-0.388^{\dagger} \\
(0.019)\end{array}$ & - \\
\hline \multicolumn{7}{|c|}{ Relationship to head (Ref: Child) } \\
\hline Partner & $\begin{array}{l}0.072 * * * \\
(0.022)\end{array}$ & $\begin{array}{l}0.069^{* * *} \\
(0.022)\end{array}$ & $\begin{array}{l}0.058^{* * *} \\
(0.022)\end{array}$ & $\begin{array}{c}0.044^{*} \\
(0.023)\end{array}$ & $\begin{array}{c}0.031 \\
(0.024)\end{array}$ & $\begin{array}{c}0.039 \\
(0.024)\end{array}$ \\
\hline Sibling & $\begin{array}{r}-0.117^{\dagger} \\
(0.016)\end{array}$ & $\begin{array}{r}-0.116^{\dagger} \\
(0.016)\end{array}$ & $\begin{array}{c}-0.115^{\dagger} \\
(0.016)\end{array}$ & $\begin{array}{c}-0.104^{\dagger} \\
(0.016)\end{array}$ & $\begin{array}{c}-0.104^{\dagger} \\
(0.016)\end{array}$ & $\begin{array}{c}-0.100^{\dagger} \\
(0.016)\end{array}$ \\
\hline Other relative & $\begin{array}{r}-0.074^{\dagger} \\
(0.022)\end{array}$ & $\begin{array}{l}-0.073 * * * \\
(0.022)\end{array}$ & $\begin{array}{r}-0.080^{\dagger} \\
(0.022)\end{array}$ & $\begin{array}{r}-0.077^{\dagger} \\
(0.022)\end{array}$ & $\begin{array}{r}-0.098^{\dagger} \\
(0.022)\end{array}$ & $\begin{array}{r}-0.105^{\dagger} \\
(0.022)\end{array}$ \\
\hline \multicolumn{7}{|c|}{ Destination (Ref: Low income country) } \\
\hline High income & $\begin{array}{c}0.110^{\dagger} \\
(0.028)\end{array}$ & - & - & - & - & - \\
\hline Upper-middle income & $\begin{array}{c}0.130^{\dagger} \\
(0.038)\end{array}$ & - & - & - & - & - \\
\hline Lower-middle income & $\begin{array}{c}-0.030 \\
(0.021)\end{array}$ & - & - & - & - & - \\
\hline \multicolumn{7}{|l|}{ Origin $\mathrm{HH}$ characteristics } \\
\hline $\ln ($ non-remittance income) & - & - & $\begin{array}{r}-0.026^{\dagger} \\
(0.003)\end{array}$ & $\begin{array}{c}-0.028^{\dagger} \\
(0.003)\end{array}$ & $\begin{array}{c}-0.025^{\dagger} \\
(0.003)\end{array}$ & $\begin{array}{c}-0.026^{\dagger} \\
(0.003)\end{array}$ \\
\hline Urban & - & - & - & $\begin{array}{c}0.010 \\
(0.018)\end{array}$ & $\begin{array}{c}0.001 \\
(0.022)\end{array}$ & $\begin{array}{c}0.011 \\
(0.023)\end{array}$ \\
\hline Number of migrants & - & - & - & $\begin{array}{r}-0.013^{\dagger} \\
(0.004)\end{array}$ & $\begin{array}{r}-0.016^{\dagger} \\
(0.004)\end{array}$ & $\begin{array}{r}-0.016^{\dagger} \\
(0.004)\end{array}$ \\
\hline $\mathrm{HH}$ head is male & - & - & - & $\begin{array}{l}-0.051^{* * *} \\
(0.018)\end{array}$ & $\begin{array}{l}-0.057^{* * *} \\
(0.017)\end{array}$ & $\begin{array}{l}-0.048^{* * *} \\
(0.018)\end{array}$ \\
\hline House is owned & - & - & - & $\begin{array}{l}0.058^{* * *} \\
(0.019)\end{array}$ & $\begin{array}{c}0.025 \\
(0.020)\end{array}$ & $\begin{array}{l}0.047^{* *} \\
(0.021)\end{array}$ \\
\hline Land is owned & - & - & - & $\begin{array}{l}0.039^{* *} \\
(0.018)\end{array}$ & $\begin{array}{c}0.033^{*} \\
(0.019)\end{array}$ & $\begin{array}{l}0.047^{* *} \\
(0.020)\end{array}$ \\
\hline Electricity & - & - & - & $\begin{array}{c}0.005 \\
(0.018)\end{array}$ & $\begin{array}{c}0.033 \\
(0.020)\end{array}$ & $\begin{array}{c}0.038^{*} \\
(0.021)\end{array}$ \\
\hline HH size & - & - & - & $\begin{array}{l}0.004^{* * *} \\
(0.001)\end{array}$ & $\begin{array}{l}0.003^{* *} \\
(0.001)\end{array}$ & $\begin{array}{c}0.003^{*} \\
(0.002)\end{array}$ \\
\hline Share of dependents & - & - & - & $\begin{array}{l}0.071 * * * \\
(0.027)\end{array}$ & $\begin{array}{l}0.065^{* *} \\
(0.027)\end{array}$ & $\begin{array}{l}0.067^{* *} \\
(0.028)\end{array}$ \\
\hline
\end{tabular}


Table 2. (Continued)

\begin{tabular}{|c|c|c|c|c|c|c|}
\hline & I & II & III & IV & $\mathrm{V}$ & VI \\
\hline Constant & $\begin{array}{c}0.393^{\dagger} \\
(0.077)\end{array}$ & $\begin{array}{c}0.352^{\dagger} \\
(0.077)\end{array}$ & $\begin{array}{c}0.566^{\dagger} \\
(0.079)\end{array}$ & $\begin{array}{c}0.516^{\dagger} \\
(0.086)\end{array}$ & $\begin{array}{c}0.562^{\dagger} \\
(0.117)\end{array}$ & $\begin{array}{c}0.176 \\
(0.119)\end{array}$ \\
\hline Destination FE & no & yes & yes & yes & yes & yes \\
\hline Origin FE & yes & yes & yes & yes & no & no \\
\hline District FE & no & no & no & no & yes & yes \\
\hline Observations & 7,536 & 7,536 & 7,536 & 7,536 & 7,536 & 7,536 \\
\hline Adj- $R^{2}$ & 0.213 & 0.217 & 0.230 & 0.240 & 0.277 & 0.225 \\
\hline
\end{tabular}

Notes: Results are obtained from OLS regressions. Standard errors in parentheses (clustered at the origin-household level). ${ }^{\dagger} \mathrm{p}<0.001 ; * * \mathrm{p}<0.01 ; * * \mathrm{p}<0.05 ;{ }^{*} \mathrm{p}<0.1$.

driven by differences in the characteristics of the households at the origin country, which are correlated with both migrants' education and their remittances. This again highlights the importance of observing both sides of the migrant-origin family relationship when analysing migrants' remittance behaviour.

With respect to other individual characteristics determining migrants' remittance behaviour, our results are largely consistent with theoretical predictions and the findings of previous literature. We find that male migrants remit more than female migrants, but this is mainly driven by the intensive margin. Moreover, married migrants are more likely to remit than unmarried migrants, while the amount remitted is uncorrelated with migrants' marital status. The amount of remittances sent further increases with both the age of the migrant and the years spent at the destination country, though at a decreasing rate. ${ }^{13}$ The latter result might be explained by two effects working against each other: on the one hand, migrants' income is likely to rise with increasing labour market experience in the destination country, leading them to remit higher amounts after having spent more time in the destination country. On the other hand, migrants' intention to return to the origin country is likely to decrease with years spent in the destination country, leading to a negative correlation between years since migration and remittances. This interpretation is in line with the results of Bollard et al. (2010), who for a sample of African migrants in OECD countries do not find a significant relationship between remittances and years spent abroad once migrants' income and their return intentions are controlled for.

Somewhat surprisingly, internal migrants are as likely to remit as international migrants. Among those remitting, however, internal migrants remit significantly lower amounts to their households left behind than international migrants. Individuals who migrated for work-related reasons are significantly more likely to remit than individuals who migrated for any other reason, while the relationship between the amount remitted and migrants' reason for migrating is less clear. In addition, migrants who have a full-time job are both more likely to remit and do remit more than migrants with a different labour force status. These results are consistent with the findings of Vanwey (2004) and Bouoiyour and Miftah (2015) and lend support to the altruism motive of remittances. They do, though, contradict the insurance motive of remittances (for example, Amuedo-Dorantes \& Pozo, 2006), which suggests that a precarious situation of the migrant should affect their transfers in a positive way.

Our results further reveal that the relationship of the migrant to the household head in the origin country is an important determinant of his or her remittance behaviour: while spouses tend to remit more than children, siblings and more distant relatives remit less, though these negative effects are only significant at the extensive margin. Lastly, our results reveal that compared to migrants residing in low-income destination countries, migrants in high-income and upper-middle-income countries are both more likely to send remittances and send higher amounts of money, conditional on remitting.

With respect to the characteristics of the households at the origin country, we find the households' non-remittance income to have a negative impact on both the probability to remit and the amount of money sent, conditional on remitting. Moreover, household size and the share of dependents at the origin household are positively correlated with migrants' remittances, while the latter effect is only significant at the extensive margin. These findings support the hypothesis that migrants from better-off 
Table 3. Determinants of the amount of remittances

\begin{tabular}{|c|c|c|c|c|c|c|}
\hline & I & II & III & IV & $\mathrm{V}$ & VI \\
\hline \multicolumn{7}{|l|}{ Migrant characteristics } \\
\hline University degree & $\begin{array}{r}0.514^{\dagger} \\
(0.087)\end{array}$ & $\begin{array}{r}0.439^{\dagger} \\
(0.086)\end{array}$ & $\begin{array}{r}0.472^{\dagger} \\
(0.084)\end{array}$ & $\begin{array}{r}0.374^{\dagger} \\
(0.079)\end{array}$ & $\begin{array}{r}0.307^{\dagger} \\
(0.082)\end{array}$ & $\begin{array}{r}0.354^{\dagger} \\
(0.083)\end{array}$ \\
\hline Male & $\begin{array}{l}0.165^{* *} \\
(0.066)\end{array}$ & $\begin{array}{l}0.173 * * * \\
(0.066)\end{array}$ & $\begin{array}{l}0.152 * * \\
(0.064)\end{array}$ & $\begin{array}{l}0.127^{* *} \\
(0.063)\end{array}$ & $\begin{array}{l}0.102^{*} \\
(0.057)\end{array}$ & $\begin{array}{l}0.122 * * \\
(0.057)\end{array}$ \\
\hline Age/100 & $\begin{array}{l}3.397^{* *} \\
(1.483)\end{array}$ & $\begin{array}{l}3.734 * * \\
(1.486)\end{array}$ & $\begin{array}{l}3.169 * * \\
(1.455)\end{array}$ & $\begin{array}{l}3.490^{* *} \\
(1.388)\end{array}$ & $\begin{array}{c}4.819^{\dagger} \\
(1.410)\end{array}$ & $\begin{array}{r}6.081^{\dagger} \\
(1.479)\end{array}$ \\
\hline $\mathrm{Age}^{2} / 100$ & $\begin{array}{c}-0.036^{* *} \\
(0.017)\end{array}$ & $\begin{array}{c}-0.040^{* *} \\
(0.017)\end{array}$ & $\begin{array}{c}-0.033 * * \\
(0.017)\end{array}$ & $\begin{array}{c}-0.035^{* *} \\
(0.016)\end{array}$ & $\begin{array}{l}-0.049 * * * \\
(0.016)\end{array}$ & $\begin{array}{c}-0.063^{\dagger} \\
(0.017)\end{array}$ \\
\hline Married & $\begin{array}{c}-0.007 \\
(0.057)\end{array}$ & $\begin{array}{c}0.002 \\
(0.057)\end{array}$ & $\begin{array}{c}-0.018 \\
(0.055)\end{array}$ & $\begin{array}{c}-0.004 \\
(0.053)\end{array}$ & $\begin{array}{c}-0.036 \\
(0.051)\end{array}$ & $\begin{array}{c}-0.025 \\
(0.051)\end{array}$ \\
\hline Years since emigration/100 & $\begin{array}{l}2.150^{* * *} \\
(0.768)\end{array}$ & $\begin{array}{l}2.222 * * * \\
(0.746)\end{array}$ & $\begin{array}{l}2.003 * * * \\
(0.685)\end{array}$ & $\begin{array}{c}2.291^{\dagger} \\
(0.695)\end{array}$ & $\begin{array}{c}2.142^{\dagger} \\
(0.619)\end{array}$ & $\begin{array}{r}2.316^{\dagger} \\
(0.665)\end{array}$ \\
\hline Years since emigration ${ }^{2} / 100$ & $\begin{array}{l}-0.054 * * * \\
(0.019)\end{array}$ & $\begin{array}{l}-0.054 * * * \\
(0.018)\end{array}$ & $\begin{array}{l}-0.047 * * * \\
(0.015)\end{array}$ & $\begin{array}{l}-0.052 * * * \\
(0.017)\end{array}$ & $\begin{array}{l}-0.045^{* * *} \\
(0.014)\end{array}$ & $\begin{array}{l}-0.047 * * * \\
(0.016)\end{array}$ \\
\hline Internal migrant & $\begin{array}{c}-0.544^{\dagger} \\
(0.069)\end{array}$ & $\begin{array}{r}-1.355^{\dagger} \\
(0.256)\end{array}$ & $\begin{array}{r}-1.337^{\dagger} \\
(0.258)\end{array}$ & $\begin{array}{r}-1.148^{\dagger} \\
(0.262)\end{array}$ & $\begin{array}{c}-0.992^{\dagger} \\
(0.259)\end{array}$ & $\begin{array}{r}-1.016^{\dagger} \\
(0.261)\end{array}$ \\
\hline \multicolumn{7}{|c|}{ Migration reason (Ref: Work related) } \\
\hline Education & $\begin{array}{c}0.154 \\
(0.100)\end{array}$ & $\begin{array}{c}0.107 \\
(0.102)\end{array}$ & $\begin{array}{c}0.134 \\
(0.090)\end{array}$ & $\begin{array}{l}0.181^{* *} \\
(0.085)\end{array}$ & $\begin{array}{c}0.094 \\
(0.084)\end{array}$ & $\begin{array}{c}0.031 \\
(0.085)\end{array}$ \\
\hline Family & $\begin{array}{c}0.137 \\
(0.093)\end{array}$ & $\begin{array}{c}0.154 \\
(0.095)\end{array}$ & $\begin{array}{c}0.161 * \\
(0.093)\end{array}$ & $\begin{array}{c}0.140 \\
(0.089)\end{array}$ & $\begin{array}{c}0.032 \\
(0.078)\end{array}$ & $\begin{array}{c}-0.107 \\
(0.079)\end{array}$ \\
\hline Other & $\begin{array}{l}-0.432 * * * \\
(0.153)\end{array}$ & $\begin{array}{l}-0.465^{* * * *} \\
(0.154)\end{array}$ & $\begin{array}{l}-0.425^{* * *} \\
(0.156)\end{array}$ & $\begin{array}{l}-0.447 * * * \\
(0.153)\end{array}$ & $\begin{array}{c}-0.474 * * * \\
(0.152)\end{array}$ & $\begin{array}{c}-0.533^{\dagger} \\
(0.155)\end{array}$ \\
\hline \multicolumn{7}{|c|}{ Labour force status (Ref: Full time employed) } \\
\hline Part time employed & $\begin{array}{c}-0.427^{\dagger} \\
(0.101)\end{array}$ & $\begin{array}{c}-0.444^{\dagger} \\
(0.100)\end{array}$ & $\begin{array}{r}-0.446^{\dagger} \\
(0.086)\end{array}$ & $\begin{array}{c}-0.422^{\dagger} \\
(0.082)\end{array}$ & $\begin{array}{c}-0.436^{\dagger} \\
(0.083)\end{array}$ & - \\
\hline Self employed & $\begin{array}{r}-0.435^{\dagger} \\
(0.062)\end{array}$ & $\begin{array}{r}-0.450^{\dagger} \\
(0.062)\end{array}$ & $\begin{array}{r}-0.456^{\dagger} \\
(0.061)\end{array}$ & $\begin{array}{r}-0.375^{\dagger} \\
(0.058)\end{array}$ & $\begin{array}{c}-0.331^{\dagger} \\
(0.055)\end{array}$ & - \\
\hline Not in labour force & $\begin{array}{r}-1.003^{\dagger} \\
(0.125)\end{array}$ & $\begin{array}{c}-0.991^{\dagger} \\
(0.125)\end{array}$ & $\begin{array}{c}-0.991^{\dagger} \\
(0.114)\end{array}$ & $\begin{array}{c}-0.947^{\dagger} \\
(0.109)\end{array}$ & $\begin{array}{r}-0.723^{\dagger} \\
(0.093)\end{array}$ & - \\
\hline \multicolumn{7}{|c|}{ Relationship to head (Ref: Child) } \\
\hline Partner & $\begin{array}{r}0.799^{\dagger} \\
(0.085)\end{array}$ & $\begin{array}{c}0.764^{\dagger} \\
(0.085)\end{array}$ & $\begin{array}{c}0.717^{\dagger} \\
(0.082)\end{array}$ & $\begin{array}{r}0.634^{\dagger} \\
(0.085)\end{array}$ & $\begin{array}{r}0.588^{\dagger} \\
(0.081)\end{array}$ & $\begin{array}{c}0.602^{\dagger} \\
(0.082)\end{array}$ \\
\hline Sibling & $\begin{array}{c}-0.032 \\
(0.060)\end{array}$ & $\begin{array}{c}-0.062 \\
(0.060)\end{array}$ & $\begin{array}{c}-0.056 \\
(0.057)\end{array}$ & $\begin{array}{c}-0.111^{*} \\
(0.057)\end{array}$ & $\begin{array}{c}-0.080 \\
(0.056)\end{array}$ & $\begin{array}{c}-0.074 \\
(0.056)\end{array}$ \\
\hline Other relative & $\begin{array}{c}-0.049 \\
(0.087)\end{array}$ & $\begin{array}{c}-0.057 \\
(0.086)\end{array}$ & $\begin{array}{c}-0.105 \\
(0.082)\end{array}$ & $\begin{array}{l}-0.148^{* *} \\
(0.075)\end{array}$ & $\begin{array}{c}-0.129^{*} \\
(0.075)\end{array}$ & $\begin{array}{c}-0.141^{*} \\
(0.075)\end{array}$ \\
\hline \multicolumn{7}{|c|}{ Destination (Ref: Low income country) } \\
\hline High income & $\begin{array}{r}1.193^{\dagger} \\
(0.113)\end{array}$ & - & - & - & - & - \\
\hline Upper-middle income & $\begin{array}{r}1.050^{\dagger} \\
(0.143)\end{array}$ & - & - & - & - & - \\
\hline Lower-middle income & $\begin{array}{r}0.272^{\dagger} \\
(0.077)\end{array}$ & - & - & - & - & - \\
\hline \multicolumn{7}{|l|}{ Origin HH characteristics } \\
\hline $\ln ($ non-remittance income) & - & - & $\begin{array}{c}-0.111^{\dagger} \\
(0.010)\end{array}$ & $\begin{array}{c}-0.138^{\dagger} \\
(0.010)\end{array}$ & $\begin{array}{c}-0.131^{\dagger} \\
(0.011)\end{array}$ & $\begin{array}{c}-0.130^{\dagger} \\
(0.011)\end{array}$ \\
\hline Urban & - & - & - & $\begin{array}{l}0.144 * * \\
(0.069)\end{array}$ & $\begin{array}{c}0.089 \\
(0.081)\end{array}$ & $\begin{array}{c}0.116 \\
(0.081)\end{array}$ \\
\hline Number of migrants & - & - & - & $\begin{array}{c}-0.064 \\
(0.016)\end{array}$ & $\begin{array}{r}-0.072^{\dagger} \\
(0.014)\end{array}$ & $\begin{array}{c}-0.074^{\dagger} \\
(0.015)\end{array}$ \\
\hline $\mathrm{HH}$ head is & - & - & - & $\begin{array}{c}-0.001 \\
(0.069)\end{array}$ & $\begin{array}{c}0.045 \\
(0.062)\end{array}$ & $\begin{array}{c}0.064 \\
(0.064)\end{array}$ \\
\hline House is owned & - & - & - & $\begin{array}{c}-0.052 \\
(0.069)\end{array}$ & $\begin{array}{c}0.053 \\
(0.071)\end{array}$ & $\begin{array}{c}0.095 \\
(0.074)\end{array}$ \\
\hline Land is owned & - & - & - & $\begin{array}{c}-0.107^{*} \\
(0.063)\end{array}$ & $\begin{array}{c}0.080 \\
(0.072)\end{array}$ & $\begin{array}{c}0.105 \\
(0.074)\end{array}$ \\
\hline Electricity & - & - & - & $\begin{array}{c}0.506^{\dagger} \\
(0.070)\end{array}$ & $\begin{array}{c}0.406^{\dagger} \\
(0.071)\end{array}$ & $\begin{array}{c}0.425^{\dagger} \\
(0.073)\end{array}$ \\
\hline HH size & - & - & - & $\begin{array}{r}0.030^{\dagger} \\
(0.005)\end{array}$ & $\begin{array}{r}0.026^{\dagger} \\
(0.005)\end{array}$ & $\begin{array}{l}0.025^{\dagger} \\
(0.005)\end{array}$ \\
\hline Share of dependents & - & - & - & $\begin{array}{c}-0.130 \\
(0.109)\end{array}$ & $\begin{array}{c}0.040 \\
(0.096)\end{array}$ & $\begin{array}{c}0.035 \\
(0.098)\end{array}$ \\
\hline
\end{tabular}


Table 3. (Continued)

\begin{tabular}{lcccccc}
\hline & I & II & III & IV & V VI \\
\hline Constant & $3.483^{\dagger}$ & $3.605^{\dagger}$ & $4.540^{\dagger}$ & $4.616^{\dagger}$ & $4.709^{\dagger}$ & $4.054^{\dagger}$ \\
& $(0.316)$ & $(0.313)$ & $(0.318)$ & $(0.324)$ & $(0.451)$ & $(0.470)$ \\
Destination FE & no & yes & yes & yes & yes & yes \\
Origin FE & yes & yes & yes & yes & no & no \\
District FE & no & no & no & no & yes & yes \\
Observations & 4,164 & 4,164 & 4,164 & 4,164 & 4,164 & 4,164 \\
Adj-R & 0.435 & 0.447 & 0.472 & 0.500 & 0.562 & 0.551 \\
\hline
\end{tabular}

Notes: Results are obtained from OLS regressions. Standard errors in parentheses (clustered at the origin-household level). ${ }^{\dagger} \mathrm{p}<0.001 ; * * \mathrm{p}<0.01 ; * * \mathrm{p}<0.05 ;{ }^{*} \mathrm{p}<0.1$.

households remit lower amounts to their families left behind. At both margins, the number of migrants from the origin household is negatively correlated with migrants' remittances. This result confirms the findings of Agarwal and Horowitz (2002) and De Brauw et al. (2013) and supports the altruism motive, but not the insurance motive of remittances.

With respect to the remaining household characteristics, we find varying effects at the extensive and at the intensive margin. Migrants from male-headed households, for example, are less likely to send remittances, while there is no such effect at the intensive margin. Consistent with the bequest motive (see, for example, Hoddinott, 1994; Melkonyan \& Grigorian, 2012), we find that households that own their house or agricultural land tend to be more likely to receive remittances, but these characteristics are uncorrelated with the amount of remittances received. ${ }^{14}$ Overall, our results reveal that origin household characteristics, particularly those related to the socio-economic status of the family, are important determinants of migrants' remittance behaviour.

\subsection{Robustness checks}

In order to examine the robustness of our main findings, we conduct a number of sensitivity checks. We do so by estimating Equation (1) for our preferred specification including the full set of individual and household controls, destination fixed effects, and origin-district fixed effects (column $\mathrm{V}$ of Tables 1-3). The main results of these robustness checks, that is, the estimated coefficients for the effect of migrants' education on remittances, are summarised in Table 4.

First, as outlined in Section 3.2, there might be an issue because the income level of the migrant cannot directly be observed in the data. As migrants' income is likely to be correlated with both their education level and their remittance behaviour, we might not be able to fully separate the (presumably positive) income effect on remittances from the effect of education on remittances. While potential solutions are limited by the data available, we check the robustness of our results by additionally controlling for migrants' occupation in the destination country, which we assume to be highly correlated with their income level. ${ }^{15}$ The respective results are displayed in Panel A of Table 4. As in our baseline results, we do not find a significant impact of migrants' education on their likelihood of sending remittances, but a positive effect on the amount of remittances for those remitting. The latter effect is slightly smaller than in our baseline regression ( 0.27 as compared to 0.31$)$, but still large and highly significant. Assuming migrants' occupation to be highly correlated with their income level, this suggests that not controlling for income does not largely affect our results.

Second, there is a concern that our findings might be driven by students, who are likely to send lower amounts of remittances to their origin households, or by individuals who migrated for the purpose of pursuing education in the destination country. This would be in line with the investment motive, which suggests that the origin household supports the migrant early in life to further his education, while the migrant starts to fulfil his part of the implicit contract only later in his life-cycle, when his income increases (see, Cox, Eser, \& Jimenez, 1998; Rapoport \& Docquier, 2006). To test whether this is driving our results, we re-estimate our model for two different samples: (i) a sample 
Table 4. Robustness checks

\begin{tabular}{llll}
\hline & Total remittances & Extensive margin & Intensive margin \\
\hline A. Controlling for migrants' occupation & &
\end{tabular}

A. Controlling for migrants' occupation

University degree

Observations

B. Sample restrictions

University degree

Observations

University degree

Observations

C. Alternative skill definition

University degree

Observations

D. Excl. single origin countries

University degree

Observations

University degree

Observations

University degree

Observations

University degree

Observations

University degree

Observations

\section{Controlling for migrants' occupation}

\begin{tabular}{ccc}
\hline $\begin{array}{c}208.115^{*} \\
(125.526)\end{array}$ & $\begin{array}{c}-0.001 \\
(0.022)\end{array}$ & $\begin{array}{c}0.269 * * * \\
(0.086)\end{array}$ \\
\hline 6,820 & 6,820 & 3,753
\end{tabular}

Excl. Students

\begin{tabular}{ccc}
\hline $\begin{array}{c}282.289 * * \\
(132.775)\end{array}$ & $\begin{array}{c}-0.010 \\
(0.024)\end{array}$ & $\begin{array}{c}0.292^{\dagger} \\
(0.084)\end{array}$ \\
\hline 7,054 & $\begin{array}{c}7,054 \\
\text { Excl. if migrated to study }\end{array}$ & \\
\hline $\begin{array}{c}325.539 * * \\
(153.012)\end{array}$ & $\begin{array}{c}-0.018 \\
(0.026)\end{array}$ & $\begin{array}{c}0.395^{\dagger} \\
(0.089)\end{array}$ \\
\hline 6,544 & 6,544 & 3,777
\end{tabular}

University or vocational education

\begin{tabular}{ccc}
\hline $\begin{array}{r}242.392^{\dagger} \\
(65.850)\end{array}$ & $\begin{array}{c}0.026 \\
(0.016)\end{array}$ & $\begin{array}{l}0.363^{\dagger} \\
(0.056)\end{array}$ \\
\hline 7,536 & 7,536 & 4,164
\end{tabular}

Excl. Burkina Faso

\begin{tabular}{ccc}
\hline $\begin{array}{c}264.594^{* *} \\
(117.982)\end{array}$ & $\begin{array}{c}-0.009 \\
(0.021)\end{array}$ & $\begin{array}{c}0.308^{\dagger} \\
(0.083)\end{array}$ \\
\hline 6,109 & $\begin{array}{c}6,109 \\
\text { Excl. Kenya }\end{array}$ & 3,467 \\
\hline
\end{tabular}

\begin{tabular}{ccc}
\hline $\begin{array}{c}243.222^{* *} \\
(120.481)\end{array}$ & $\begin{array}{c}0.032 \\
(0.025)\end{array}$ & $\begin{array}{c}0.234^{* *} \\
(0.113)\end{array}$ \\
\hline 6,093 & 6,093 & 3,352
\end{tabular}

Excl. Nigeria

\begin{tabular}{ccc}
\hline $\begin{array}{c}258.311^{* *} \\
(128.295)\end{array}$ & $\begin{array}{c}-0.021 \\
(0.025)\end{array}$ & $\begin{array}{c}0.396^{\dagger} \\
(0.096)\end{array}$ \\
\hline 5,493 & $\begin{array}{c}5,493 \\
\text { Excl. Senegal }\end{array}$ & 3,015 \\
\hline $\begin{array}{c}\text { 314.906** } \\
(134.807)\end{array}$ & $\begin{array}{c}0.003 \\
(0.024)\end{array}$ & $\begin{array}{c}0.316^{\dagger} \\
(0.090)\end{array}$ \\
\hline 5,861 & $\begin{array}{c}5,861 \\
\text { Excl. Uganda }\end{array}$ & 3,011 \\
\hline $\begin{array}{c}-0.027 \\
(138.636)\end{array}$ & $(0.024)$ & $\begin{array}{c}0.257^{* * *} \\
(0.086)\end{array}$ \\
\hline 6,588 & 6,588 & 3,811 \\
\hline
\end{tabular}


Table 4. (Continued)

\begin{tabular}{lccc}
\hline & Total remittances & Extensive margin & Intensive margin \\
\hline E. Alternative estimation methods & Tobit & Probit & Heckman \\
\cline { 2 - 4 } & $\begin{array}{l}318.227^{*} \\
(162.967)\end{array}$ & $\begin{array}{c}-0.006 \\
(0.022)\end{array}$ & $\begin{array}{c}\text { (0.336 } \\
\text { University degree }\end{array}$ \\
\cline { 2 - 4 } & - & - & $0.072)$ \\
Inverse mills ratio & 7,536 & 7,477 & 0.318 \\
Observations & & $0.232)$ \\
\hline
\end{tabular}

Notes: All results are obtained from regressions of Equation (1) for our preferred specification including a full set of individual and household controls, destination fixed effects, and origin-district fixed effects (column $\mathrm{V}$ of Table 1-3). Panels A to D show coefficients of OLS regressions. Panel E shows coefficients of a Tobit regression, average marginal effects of a Probit regression, and coefficients of the outcome equation of a Heckman selection model (the results of the selection equation are similar to the Probit results). Standard errors in parentheses (clustered at the origin-household level). ${ }^{\dagger} \mathrm{p}<0.001 ; * * \mathrm{p}<0.01 ; * * \mathrm{p}<0.05 ;{ }^{*} \mathrm{p}<0.1$.

that excludes migrants who are still in education and (ii) a sample that excludes individuals who migrated in order to pursue education. The respective results are shown in Panel B of Table 4. For both samples, our previous findings hold. At the intensive margin, the coefficient for migrants' university degree slightly increases once individuals who migrated in order to pursue education are excluded, but is overall stable in significance and magnitude.

Third, we check whether our results are sensitive to the definition of the skill level of the migrant. Specifically, we estimate an alternative specification in which we define high-skilled migrants as those who have obtained a university degree or a vocational education degree. The respective estimates using this broader definition of high-skilled migrants are presented in Panel $\mathrm{C}$ of Table 4. At the intensive margin, the coefficient for migrants' education remains similar in both magnitude and significance. At the extensive margin, however, we now find a significantly positive (at the $10 \%$ level), though small, effect of migrants' education on their probability to remit, suggesting that migrants with a vocational degree are slightly more likely to remit than migrants with a lower educational qualification.

Fourth, there might be a concern that our results do not hold for all origin countries, but are driven by a single country. We therefore successively exclude one origin country from the sample and estimate Equation (1) based on the remaining four origin countries. ${ }^{16}$ The results of these five regressions are shown in Panel D of Table 4. While the size of the positive effect of education on the amount of remittances sent slightly varies over the different countries, our results are overall robust and confirm that our estimates are not driven by a single origin country.

Lastly, we check whether our findings are robust to the estimation method chosen to generate our results. In our baseline specification, we estimate our model using OLS for all outcomes. While this is an obvious choice for the intensive margin of migrants' remittances, the censored nature of the total amount of remittances and the binary nature of the probability to remit might call for the use of a nonlinear estimation method. In Panel E of Table 4, we therefore use a Tobit model to estimate the total amount of remittances and a Probit model to estimate the probability to remit. The results are robust to this alternative model choice in terms of both significance and magnitude. ${ }^{17}$

In addition, when analysing the intensive margin of remittances, we have implicitly assumed that the choice of how much to remit is independent of the decision to remit. However, this is not necessarily the case and hence migrants who remit may be a non-random sample of the migrants' population. To address this issue, we alternatively use a selection model as proposed by Heckman (1976) to correct for selection in the remittances equation. As is well known, this model generally requires an exclusion restriction, that is, at least one variable that affects selection but not the outcome 
of interest. However, as finding a valid exclusion restriction is difficult to achieve in our context, identification in our model crucially relies on the nonlinearity of the selection bias. The results of the second stage of the Heckman selection model are shown in the third column of Panel E. With a coefficient of 0.34 , the estimated effect of having a university degree is only slightly higher than in the baseline regression (0.31), so the results are robust to this alternative specification. Also, the inverse mills ratio, which is the covariance between the errors of the selection and the outcome equation, is not statistically significant, suggesting that selection into remitting is of minor relevance in our context. ${ }^{18}$

\subsection{Heterogeneous effects across migrants' destination countries}

In Section 4.1, we have shown that migrants living in wealthier destination countries have a higher probability of sending remittances and send larger amounts of money to their households left behind. Moreover, internal migrants appear to send lower amounts than international migrants (Tables 1-3). To gain further insights into the heterogeneous effects of migrants' education on their remittance behaviour in different destination countries, we split the baseline sample into three sub-samples: (i) migrants in OECD destination countries, (ii) migrants in non-OECD destination countries, and (iii) internal migrants.

Although OECD migrants are more likely to remit and remit higher amounts than non-OECD and internal migrants (see Table B1 in the Supplementary Materials), the money sent by the latter two groups makes up for a large proportion of the total level of remittances received by the origin countries. This is illustrated in Figure 2, which displays the amount of remittances received from (i) OECD migrants, (ii) non-OECD migrants, and (iii) internal migrants as a share of the total remittances received by all households in the respective origin country. Remittances received from non-OECD and internal migrants make up for 35 to 47 per cent of the total level of remittances received by households in Kenya, Nigeria, Senegal, and Uganda, and even account for 90 per cent of the total amount of remittances in Burkina Faso. This again highlights the importance of investigating the remittance behaviour of all migrants, and not restricting the analysis to migrants in OECD countries.

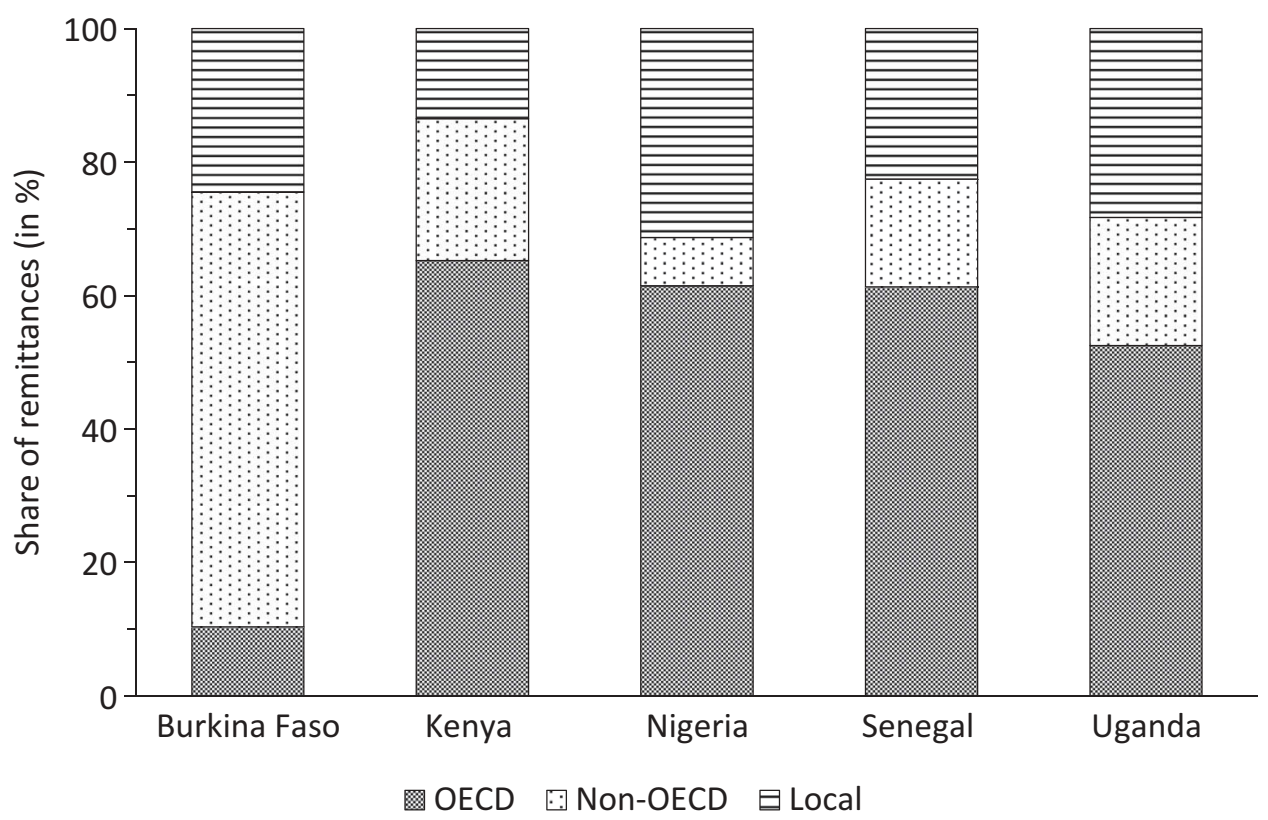

Figure 2. Share of the total level of remittances received by destination country. 
Table 5. Effect of education on remittances by destination country

\begin{tabular}{|c|c|c|c|c|c|c|}
\hline & I & II & III & IV & $\mathrm{V}$ & VI \\
\hline A. Extensive margin & \multicolumn{6}{|c|}{ OECD countries } \\
\hline University degree & $\begin{array}{l}-0.042 \\
(0.036)\end{array}$ & $\begin{array}{l}-0.049 \\
(0.036)\end{array}$ & $\begin{array}{l}-0.035 \\
(0.034)\end{array}$ & $\begin{array}{l}-0.022 \\
(0.034)\end{array}$ & $\begin{array}{l}-0.053 \\
(0.034)\end{array}$ & $\begin{array}{l}-0.039 \\
(0.037)\end{array}$ \\
\hline Observations & \multicolumn{6}{|c|}{$\begin{array}{c}1,589 \\
\text { Non-OECD countries }\end{array}$} \\
\hline University degree & $\begin{array}{l}-0.068 \\
(0.052)\end{array}$ & $\begin{array}{l}-0.028 \\
(0.053)\end{array}$ & $\begin{array}{l}-0.019 \\
(0.053)\end{array}$ & $\begin{array}{l}-0.001 \\
(0.054)\end{array}$ & $\begin{array}{l}-0.007 \\
(0.065)\end{array}$ & $\begin{array}{c}0.008 \\
(0.068)\end{array}$ \\
\hline Observations & \multicolumn{6}{|c|}{$\begin{array}{c}1,663 \\
\text { Internal migrants }\end{array}$} \\
\hline University degree & $\begin{array}{l}-0.024 \\
(0.032)\end{array}$ & $\begin{array}{l}-0.024 \\
(0.032)\end{array}$ & $\begin{array}{l}-0.008 \\
(0.031)\end{array}$ & $\begin{array}{c}0.006 \\
(0.030)\end{array}$ & $\begin{array}{l}-0.002 \\
(0.030)\end{array}$ & $\begin{array}{c}0.039 \\
(0.030)\end{array}$ \\
\hline $\begin{array}{l}\text { Observations } \\
\text { B. Intensive margin }\end{array}$ & & & OEC & Intries & & \\
\hline University degree & $\begin{array}{c}0.209 \\
(0.127)\end{array}$ & $\begin{array}{l}0.216^{*} \\
(0.127)\end{array}$ & $\begin{array}{l}0.233^{*} \\
(0.122)\end{array}$ & $\begin{array}{c}0.174 \\
(0.120)\end{array}$ & $\begin{array}{c}0.095 \\
(0.134)\end{array}$ & $\begin{array}{c}0.110 \\
(0.133)\end{array}$ \\
\hline Observations & \multicolumn{6}{|c|}{$\begin{array}{c}1,111 \\
\text { Non-OECD countries }\end{array}$} \\
\hline University degree & $\begin{array}{c}0.827 * * * \\
(0.270)\end{array}$ & $\begin{array}{l}0.541 * * \\
(0.268)\end{array}$ & $\begin{array}{l}0.595 * * \\
(0.269)\end{array}$ & $\begin{array}{l}0.609 * * \\
(0.266)\end{array}$ & $\begin{array}{c}0.787 * * * \\
(0.301)\end{array}$ & $\begin{array}{c}0.850 * * * \\
(0.311)\end{array}$ \\
\hline Observations & \multicolumn{6}{|c|}{$\begin{array}{c}902 \\
\text { Internal migrants }\end{array}$} \\
\hline University degree & $\begin{array}{l}0.620^{\dagger} \\
(0.121)\end{array}$ & $\begin{array}{l}0.620^{\dagger} \\
(0.121)\end{array}$ & $\begin{array}{l}0.668^{\dagger} \\
(0.119)\end{array}$ & $\begin{array}{l}0.530^{\dagger} \\
(0.109)\end{array}$ & $\begin{array}{l}0.394^{\dagger} \\
(0.113)\end{array}$ & $\begin{array}{l}0.460^{\dagger} \\
(0.114)\end{array}$ \\
\hline Observations & \multicolumn{6}{|c|}{2,151} \\
\hline Individual Controls & all & all & all & all & all & no LFS \\
\hline $\mathrm{HH}$ Controls & no & no & income & all & all & all \\
\hline Destination FE & no & yes & yes & yes & yes & yes \\
\hline Origin FE & yes & yes & yes & yes & no & no \\
\hline District FE & no & no & no & no & yes & yes \\
\hline
\end{tabular}

Notes: Results are obtained from OLS regressions. Standard errors in parentheses (clustered at the origin-household level). ${ }^{\dagger} \mathrm{p}<0.001 ; * * * \mathrm{p}<0.01 ;{ }^{*} \mathrm{p}<<0.05 ;{ }^{*} \mathrm{p}<0.1$.

Based on estimating Equation (1), Table 5 reports the coefficients of our variable of interest, the estimated effect of migrants' education on the extensive and intensive margin of remittances, separately for the three different destination groups. ${ }^{19}$ In line with our previously presented results, the estimated effect of having a university degree on the likelihood of sending remittances is small and not statistically significant across all specifications for the three destination groups (Panel A). This reveals that, irrespective of their destination, migrants with a university degree do not have a higher probability of sending remittances than migrants without a university degree.

At the intensive margin (Panel B), we find evidence that conditional on sending remittances, highskilled migrants in non-OECD countries and internal migrants send larger amounts of money compared to less skilled migrants. In our preferred specification (column V), the estimated education effect is 0.79 and 0.39 , respectively, suggesting that high-skilled individuals that migrate to a nonOECD country remit about 79 per cent and high-skilled individuals that migrate internally remit about 39 per cent more than comparable migrants without a university degree. Given the average amount of 
remittances sent by these groups, high-skilled migrants in non-OECD countries remit around 574 USD and high-skilled internal migrants remit about 174 USD more than comparable low-skilled migrants in these countries.

The results for migrants in OECD countries, in contrast, differ from the findings of previous literature. The estimated effect of having a university degree is positive, but substantially smaller than the estimated effects for the other two destination groups. In addition, the estimated coefficient decreases further and becomes insignificant once the full set of household characteristics and district fixed effects (columns IV-VI) are controlled for. According to these results, high-skilled migrants living in OECD countries do not send more remittances than comparable low-skilled migrants in these countries.

One reasonable explanation for this pattern might be migrants' educational downgrading in destination countries (Dustmann \& Glitz, 2011). In OECD countries, with their high educational standards, the education level of migrants might not be fully recognised in the labour market. Therefore, in the destination country, high-skilled migrants may work in jobs that require lower qualifications than the jobs they held in their home country, and they may thus work in similar jobs as lower skilled migrants. This conjecture is supported by our data: in OECD countries, migrants with a university degree show only a 35 per cent higher probability of working in a high-skilled job than migrants without a university degree. Compared to the figures for migrants in non-OECD countries $(222 \%)$ and internal migrants $(71 \%)$, this is a particular low probability, supporting the existence of a strong educational downgrading of migrants in OECD destination countries. ${ }^{20}$ In the OECD labour markets, migrants' returns to having a university degree obtained in Sub-Saharan Africa are therefore likely to be small and thus do not affect their amount of remittances sent. ${ }^{21}$ This has important implications for migrants' origin countries: if substantial public and private investments in higher education are devoted to individuals who work abroad in jobs that do not correspond to their educational qualifications, then the potential gain from high-skilled emigration, in terms of higher remittances due to higher foreign wages, is undermined by the waste of migrants' human capital.

\section{Conclusion}

The so-called 'brain-drain' or the migration of high-skilled individuals to other regions where human capital is abundant is a major concern for many developing countries, as it represents the loss of their most talented workers. While remittances may offset some of the negative externalities of the brain drain, there is also a concern that highly educated migrants may send less remittances to their households at the origin country, suggesting that an increase in high-skill emigration will lower remittance flows (for example, Adams, 2009; Faini, 2007; Niimi et al., 2010).

This paper investigates the relationship between migrants' education and remittances using unique microdata from five countries in Sub-Saharan Africa. In contrast to the few existing studies using microdata, which are based on household surveys collected in migrants' destination countries, the data used in this study includes detailed information on both the migrants and the households at the origin country. In addition, the data enables us to not only restrict our analysis to migrants in OECD destination countries, but to further provide important insights into the remitting behaviour of internal migrants and migrants in non-OECD destination countries, which have so far been neglected by the existing literature.

Overall, we find that migrants with a university degree remit about 265-362 USD more to their origin households than migrants without a university degree. This effect, however, is mainly driven by the intensive margin: while migrants' education does not affect the likelihood of sending remittances, it has a sizable positive effect on the amount of remittances sent for those who are remitting. In our preferred specification, migrants with a university degree remit around 31 per cent or 307 USD more than migrants without a university degree. This positive effect of education on the amount of remittances sent is robust to a number of sensitivity analyses. This contradicts the finding of previous studies based on macrodata and suggests that some of the negative externalities of the brain drain on origin countries can be counterbalanced by migrants' remittances. 
When splitting the sample into migrants in OECD countries, migrants in non-OECD countries, and internal migrants, the results remain robust at the extensive margin. For migrants in non-OECD countries and internal migrants the results also remain robust at the intensive margin. For migrants in OECD countries, however, the positive effect of migrants' education on the amount of remittances sent vanishes once characteristics of the household at the origin country are controlled for. This reveals that part of the positive effect of migrants' education on remittances is due to unobserved heterogeneity across origin households and thus highlights the importance of controlling for characteristics of both sides of the migrant-household relationship when analysing migrants' remittance behaviour. Furthermore, this result supports previous findings of migrants' educational downgrading in destination countries (see, for example, Mattoo, Neagu, \& Özden, 2008). In OECD countries, migrants' returns to having a university degree obtained in Sub-Saharan Africa are likely to be small, as they are more likely to work in a lower skilled job, such that their educational qualification is uncorrelated with the amount of remittances sent.

Although our results do not necessarily reflect causal effects, they provide important insights from a policy perspective. In particular, two policy implications can be drawn from our analysis: first, highskilled migrants do not remit less than low-skilled migrants as emphasised by part of the existing literature. In general, high-skilled migrants have higher earnings and fewer liquidity constraints than low-skilled migrants and in turn send more remittances. This suggests that policies that favour skilled migration are beneficial for the amount of remittances received by the origin country. The conclusion of previous studies based on macrodata, that remittances will fall as the migrant skill level rises, is thus not supported by our findings. Second, policy-makers should focus on implementing policies that increase the probability of sending remittances for both high- and low-skilled migrants. One promising measure to achieve this goal is to strengthen cooperation between origin and destination countries, both in terms of information sharing about potential needs in the destination country's labour market as well as in terms of improving the recognition of foreign qualifications (Mattoo et al., 2008), which will improve the economic situation of migrants in their destination country and therefore likely lead to higher remittances flows to their countries of origin.

\section{Acknowledgements}

The authors are grateful to Thomas Bauer, the editor Emmanuel Teitelbaum, two anonymous referees as well as participants at the 2016 Development Economics and Policy Conference in Heidelberg, the ESPE 2016 in Berlin, the RGS/RWI Workshop on the Economics of Migration in Essen, and the 2017 AEA meetings in Chicago for helpful comments and suggestions. We are also very thankful to the African Development Bank and the World Bank as part of the Africa Migration Project for providing most of the data. Official data and codes used in this article are available from the authors upon request.

\section{Disclosure statement}

No potential conflict of interest was reported by the authors.

\section{Notes}

1. Recent literature not only considers the negative consequences of high-skilled migration, but also points to its positive effects, suggesting the existence of a brain gain instead of a brain drain (see, for example, Batista, Lacuesta, \& Vicente, 2012; Beine, Docquier, \& Oden-Defoort, 2011; Beine, Docquier, \& Rapoport, 2001; Clemens, 2011; Dustmann, Fadlon, \& Weiss, 2011).

2. Clemens and Pettersson (2008) and Bhargava, Docquier, and Moullan (2011) provide data on the emigration of professionals in the medical sector. Regional comparisons reveal that the medical brain drain is highest in Sub-Saharan Africa (with average rates above $20 \%$ compared to $13 \%$ in South Asia and less than $10 \%$ in the other regions).

3. See Rapoport and Docquier (2006), Gibson and McKenzie (2011), Yang (2011), and Docquier and Rapoport (2012) for a general literature overview and Azam and Gubert (2006) for a review on the remittance behaviour of African migrants. 
4. Notable exceptions include Osili (2007), using a matched sample of Nigerian migrants residing in Chicago and their origin households, as well as Duval and Wolff (2010) and Bouoiyour and Miftah (2015), using household surveys from Albania and Morocco, respectively. However, these studies are different from ours as they do not address the brain drain, but focus on other aspects of migrants' remittance behaviour.

5. Empirical evidence shows, for example, that remittances increase when the household left behind experiences a negative income shock (see, for example, Yang \& Choi, 2007; Gröger \& Zylberberg, 2016; Bettin, Presbitero, \& Spatafora, 2017; Bettin \& Zazzaro, 2017).

6. According to migration statistics provided by the World Bank (2013), only 27 per cent of Sub-Saharan African migrants go to OECD countries, while the majority remain within the region (65\%) or migrate to other non-OECD destination countries $(8 \%)$.

7. While international remittances have become a more frequently researched topic in recent years, studies on internal remittances are almost non-existent. Notable exceptions are Regmi and Tisdell (2002) and De Brauw et al. (2013) analysing the remittance motives of internal migrants in Nepal and Ethiopia, respectively and De Weerdt and Hirvonen (2016) investigating, more generally, the risk sharing behaviour of internal migrants and their extended family network in Tanzania.

8. We check the robustness of our results to using alternative estimation methods, such as Tobit or Probit models (see Section 4.2).

9. The surveys are nationally representative for Nigeria, Senegal, and Uganda. In the case of Burkina Faso and Kenya, they are representative for the 10 largest provinces and the top 17 districts with the highest concentration of migrants, respectively. For further information about the data collection, see Plaza et al. (2011).

10. Note that we exclude five observations from the sample because the reported amount of yearly remittances was implausibly high, exceeding 80,000 USD.

11. Using consumption expenditures as a measure of current and long-term household welfare is standard in the literature (see, for example, Jena, 2017).

12. Descriptive statistics of all variables, separately for the samples of high- and low-skilled migrants, are shown in Table A1 in the Appendix.

13. In our preferred specification (column V), migrants' remittances peak at age 55 after having spent about 18 years in the destination country.

14. The bequest motive suggests that migrants continually send remittances to the household left behind to strengthen the relationship and thereby insure future bequests. Therefore, it predicts a positive correlation between the wealth of the origin household and migrants' probability to remit, but no strong relationship between the household's wealth and the amount sent by the migrants.

15. As occupational information is not available for all migrants in our sample, we refrain from using this variable in our baseline regressions.

16. Due to the relatively small sample sizes, we refrain from estimating the regressions separately for each origin country.

17. Note that in the Probit model, the sample size is slightly reduced, because there exist a few districts in which no or all migrants remit, which have to be excluded from the sample.

18. Note, however, that this finding may partly be due to the weak identification problem inherited in selection models without exclusion restrictions. Note also that the Heckman model assumes that all non-remitters are unwilling to send money back home. However, as Bettin, Lucchetti, \& Zazzaro (2012) argue, in the presence of transfer costs and budget constraints, some migrants might also be prevented from remitting. In such a case, the appropriate framework to model the remittance decisions is a double-hurdle model, which features both censoring and selection mechanisms. However, as the censoring point, that is, the minimum amount of remittances below which the costs of remitting are not offset by the additional utility of remitting, is unknown, we refrain from utilising a double-hurdle model in our study.

19. The results for the overall effect are shown in Table B2 in the Supplementary Materials.

20. Information on the skill level of migrants' jobs is obtained from self-reported information on migrants' occupations in the destination countries, which is only available for a sub-sample of all migrants in our sample.

21. In our sample, high-skilled migrants in OECD countries remit similar amounts as high-skilled migrants in non-OECD countries (about 3000 USD per year), while low-skilled migrants remit more than three times the amount in OECD countries than in non-OECD countries (2100 versus 600 USD per year).

\section{References}

Adams, R. H. (2009). The determinants of international remittances in developing countries. World Development, 37, $93-103$. doi:10.1016/j.worlddev.2007.11.007

Agarwal, R., \& Horowitz, A. W. (2002). Are international remittances altruism or insurance? Evidence from Guyana using multiple-migrant households. World Development, 30, 2033-2044. doi:10.1016/S0305-750X(02)00118-3

Amuedo-Dorantes, C., \& Pozo, S. (2006). Remittances as insurance: Evidence from Mexican immigrants. Journal of Population Economics, 19, 227-254. doi:10.1007/s00148-006-0079-6

Azam, J.-P., \& Gubert, F. (2006). Migrants' remittances and the household in Africa: A review of evidence. Journal of African Economies, 15, 426-462. doi:10.1093/jae/ej1030 
Batista, C., Lacuesta, A., \& Vicente, P. C. (2012). Testing the 'Brain Gain' hypothesis: Micro evidence from Cape Verde. Journal of Development Economics, 97, 32-45. doi:10.1016/j.jdeveco.2011.01.005

Beine, M., Docquier, F., \& Oden-Defoort, C. (2011). A panel data analysis of the brain gain. World Development, 39, 523-532. doi:10.1016/j.worlddev.2010.03.009

Beine, M., Docquier, F., \& Rapoport, H. (2001). Brain drain and economic growth: Theory and evidence. Journal of Development Economics, 64, 275-289. doi:10.1016/S0304-3878(00)00133-4

Beine, M., Docquier, F., \& Rapoport, H. (2008). Brain drain and human capital formation in developing countries: Winners and losers. Economic Journal, 118, 631-652. doi:10.1111/j.1468-0297.2008.02135.x

Bettin, G., Lucchetti, R., \& Zazzaro, A. (2012). Endogeneity and sample selection in a model for remittances. Journal of Development Economics, 99, 370-384. doi:10.1016/j.jdeveco.2012.05.001

Bettin, G., Presbitero, A. F., \& Spatafora, N. L. (2017). Remittances and vulnerability in developing countries. World Bank Economic Review, 31, 1-23.

Bettin, G., \& Zazzaro, A. (2017). The impact of natural disasters on remittances to low-and middle-income countries. Journal of Development Studies, 54, 481-500. doi:10.1080/00220388.2017.1303672

Bhargava, A., Docquier, F., \& Moullan, Y. (2011). Modeling the effects of physician emigration on human development. Economics \& Human Biology, 9, 172-183. doi:10.1016/j.ehb.2010.12.004

Bollard, A., McKenzie, D., \& Morten, M. (2010). The remitting patterns of African migrants in the OECD. Journal of African Economies, 19, 605-634. doi:10.1093/jae/ejq031

Bollard, A., McKenzie, D., Morten, M., \& Rapoport, H. (2011). Remittances and the brain drain revisited: The microdata show that more educated migrants remit more. World Bank Economic Review, 25, 132-156. doi:10.1093/wber/lhr013

Bouoiyour, J., \& Miftah, A. (2015). Why do migrants remit? Testing hypotheses for the case of Morocco. IZA Journal of Migration, 4, 1-20. doi:10.1186/s40176-014-0027-2

Brücker, H., Capuano, S., \& Marfouk, A. (2013). Education, gender and international migration: Insights from a panel-dataset 1980-2010. Retrieved from http://www.iab.de/en/daten/iab-brain-drain-data.aspx

Clemens, M. A. (2011). Economics and emigration: Trillion-dollar bills on the sidewalk? Journal of Economic Perspectives, 25 (3), 83-106. doi:10.1257/jep.25.3.83

Clemens, M. A., \& McKenzie, D. (2018). Why don’t remittances appear to affect growth? The Economic Journal. doi:10.1111/ ecoj. 12463.

Clemens, M. A., \& Pettersson, G. (2008). New data on African health professionals abroad. Human Resources for Health, 6(1), 1-11. doi:10.1186/1478-4491-6-1

Cox, D., Eser, Z., \& Jimenez, E. (1998). Motives for private transfers over the life cycle: An analytical framework and evidence for Peru. Journal of Development Economics, 55, 57-80. doi:10.1016/S0304-3878(97)00056-4

De Brauw, A., Mueller, V., \& Woldehanna, T. (2013). Motives to remit: Evidence from tracked internal migrants in Ethiopia. World Development, 50, 13-23. doi:10.1016/j.worlddev.2013.04.008

De Weerdt, J., \& Hirvonen, K. (2016). Risk sharing and internal migration. Economic Development and Cultural Change, 65, 63-86. doi:10.1086/687577

Di Maria, C., \& Lazarova, E. A. (2012). Migration, human capital formation, and growth: An empirical investigation. World Development, 40, 938-955. doi:10.1016/j.worlddev.2011.11.011

Djiofack, C. Z., Djimeu, E. W., \& Boussichas, M. (2013). Impact of qualified worker emigration on poverty: A macro-microsimulation approach for an African economy. Journal of African Economies, 42, 817-828.

Docquier, F., \& Rapoport, H. (2012). Globalization, brain drain, and development. Journal of Economic Literature, 50, 681-730. doi:10.1257/jel.50.3.681

Docquier, F., Rapoport, H., \& Salomone, S. (2012). Remittances, migrants' education and immigration policy: Theory and evidence from bilateral data. Regional Science and Urban Economics, 23(1), 1-52.

Dustmann, C., Fadlon, I., \& Weiss, Y. (2011). Return migration, human capital accumulation and the brain drain. Journal of Development Economics, 95, 58-67. doi:10.1016/j.jdeveco.2010.04.006

Dustmann, C., \& Glitz, A. (2011). Migration and education. In E. A. Hanushek, S. Machin, \& L. Woessmann (Eds.), Handbook of the economics of education (Vol. 4, pp. 327-439). Amsterdam: Elsevier.

Dustmann, C., \& Mestres, J. (2010). Remittances and temporary migration. Journal of Development Economics, 92, 62-70. doi:10.1016/j.jdeveco.2008.12.002

Duval, L., \& Wolff, F.-C. (2010). Remittances matter: Longitudinal evidence from Albania. Post-Communist Economies, 22, 7397. doi:10.1080/14631370903525611

Faini, R. (2007). Remittances and the brain drain: Do more skilled migrants remit more? World Bank Economic Review, 21, 177191. doi:10.1093/wber/lhm006

George, J., Dei, S., \& Asgharzadeh, A. (2002). What is to be done? A look at some causes and consequences of the African Brain drain. African Issues, 30(1), 31-36. doi:10.2307/1167087

Gibson, J., \& McKenzie, D. (2011). Eight questions about brain drain. Journal of Economic Perspectives, 25(3), $107-128$. doi:10.1257/jep.25.3.107

Gibson, J., \& McKenzie, D. (2012). The economic consequences of 'Brain Drain' of the best and brightest: Microeconomic evidence from five countries. The Economic Journal, 122, 339-375. doi:10.1111/ecoj.2012.122.issue-560

Gröger, A., \& Zylberberg, Y. (2016). Internal labor migration as a shock coping strategy: Evidence from a Typhoon. American Economic Journal: Applied Economics, 8(2), 123-153. 
Haque, N. U., \& Kim, S.-J. (1995). 'Human Capital Flight': Impact of migration on income and growth. Staff Papers International Monetary Fund, 42, 577-607. doi:10.2307/3867533

Heckman, J. J. (1976). The common structure of statistical models of truncation, sample selection and limited dependent variables and a simple estimator for such models. Annals of Economic and Social Measurement, 5(4), 475-492.

Hoddinott, J. (1994). A model of migration and remittances applied to Western Kenya. Oxford Economic Papers, 46, 459-476. doi:10.1093/oxfordjournals.oep.a042141

Jena, F. (2017). Migrant remittances and physical investment purchases: Evidence from Kenyan households. Journal of Development Studies, 54, 312-326.

Le Goff, M., \& Salomone, S. (2016). Remittances and the changing composition of migration. The World Economy, 39, 513529. doi:10.1111/twec.2016.39.issue-4

Licuanan, V., Mahmoud, T. O., \& Steinmayr, A. (2015). The drivers of diaspora donations for development: Evidence from the Philippines. World Development, 65, 94-109. doi:10.1016/j.worlddev.2014.05.010

Mattoo, A., Neagu, I. C., \& Özden, Ç. (2008). Brain waste? Educated immigrants in the US labor market. Journal of Development Economics, 87, 255-269. doi:10.1016/j.jdeveco.2007.05.001

Melkonyan, T. A., \& Grigorian, D. A. (2012). Microeconomic implications of remittances in an overlapping generations model with altruism and a motive to receive inheritance. Journal of Development Studies, 48, 1026-1044. doi:10.1080/ 00220388.2011 .598507

Niimi, Y., Özden, C., \& Schiff, M. (2010). Remittances and the brain drain: Skilled migrants do remit less. Annals of Economics and Statistics, 97/98, 123-141. doi:10.2307/41219112

Osili, U. O. (2007). Remittances and savings from international migration: Theory and evidence using a matched sample. Journal of Development Economics, 83, 446-465. doi:10.1016/j.jdeveco.2006.06.003

Plaza, S., Navarrete, M., \& Ratha, D. (2011). Migration and remittances household surveys in Sub-Saharan Africa: Methodological aspects and main findings. Washington, DC: World Bank.

Rapoport, H., \& Docquier, F. (2006). The economics of migrants' remittances. In S.-C. Kolm \& J. Mercier Ythier (Eds.), Handbook of the economics of giving, altruism and reciprocity (Vol. 2, pp. 1135-1198). Amsterdam: Elsevier.

Regmi, G., \& Tisdell, C. (2002). Remitting behaviour of nepalese rural-to-urban migrants: Implications for theory and policy. Journal of Development Studies, 38, 76-94. doi:10.1080/00220380412331322351

UNESCO. (2016). Educational attainment of the population aged 25 years and older. Retrieved from http://www.uis.unesco.org/ Education/Pages/educational-attainment-data-release.aspx

Vanwey, L. K. (2004). Altruistic and contractual remittances between male and female migrants and households in Rural Thailand. Demography, 41, 739-756. doi:10.1353/dem.2004.0039

World Bank. (2013). Bilateral migration matrix 2013. Retrieved from http://www.worldbank.org/en/topic/migrationremittances diasporaissues/brief/migration-remittances-data

World Bank. (2015). World bank country and lending groups. Retrieved from http://data.worldbank.org/about/country-andlending-groups

Yang, D. (2011). Migrant remittances. Journal of Economic Perspectives, 25(3), 129-152. doi:10.1257/jep.25.3.129

Yang, D., \& Choi, H. (2007). Are remittances insurance? Evidence from rainfall shocks in the Philippines. The World Bank Economic Review, 21, 219-248. doi:10.1093/wber/lhm003 


\section{Appendix}

Table A1. Descriptive statistics

\begin{tabular}{|c|c|c|c|c|c|c|c|}
\hline & \multicolumn{2}{|c|}{ All migrants } & \multicolumn{2}{|c|}{$\begin{array}{l}\text { University } \\
\text { degree }\end{array}$} & \multicolumn{2}{|c|}{ No degree } & \multirow[b]{2}{*}{$\Delta$ Mean } \\
\hline & Mean & S.D. & Mean & S.D. & Mean & S.D. & \\
\hline \multicolumn{8}{|l|}{ Migrant's characteristics } \\
\hline Probability of remitting & 0.553 & 0.497 & 0.551 & 0.498 & 0.553 & 0.497 & -0.002 \\
\hline Total remittances (in 1,000 USD) & 0.547 & 1.872 & 1.114 & 3.092 & 0.491 & 1.693 & $0.623^{\dagger}$ \\
\hline Remittances, cond. on remitting (in 1,000 USD) & 0.991 & 2.430 & 2.021 & 3.941 & 0.888 & 2.198 & $1.133^{\dagger}$ \\
\hline University degree & 0.091 & 0.288 & - & - & - & - & - \\
\hline Male & 0.720 & 0.449 & 0.666 & 0.472 & 0.725 & 0.446 & $-0.059 * * *$ \\
\hline Age & 34.944 & 9.582 & 34.685 & 9.606 & 34.970 & 9.580 & -0.285 \\
\hline Married & 0.661 & 0.473 & 0.605 & 0.489 & 0.667 & 0.471 & $-0.062 * * *$ \\
\hline Years since emigration & 7.520 & 7.328 & 7.159 & 7.440 & 7.556 & 7.316 & -0.397 \\
\hline Internal migrant & 0.568 & 0.495 & 0.459 & 0.499 & 0.579 & 0.494 & $-0.120^{\dagger}$ \\
\hline \multicolumn{8}{|l|}{ Migration reason } \\
\hline Education & 0.132 & 0.338 & 0.264 & 0.441 & 0.118 & 0.323 & $0.145^{\dagger}$ \\
\hline Work & 0.687 & 0.464 & 0.605 & 0.489 & 0.696 & 0.460 & $-0.091^{\dagger}$ \\
\hline Family & 0.152 & 0.359 & 0.112 & 0.316 & 0.156 & 0.363 & $-0.044 * * *$ \\
\hline Other & 0.029 & 0.168 & 0.019 & 0.136 & 0.030 & 0.170 & -0.011 \\
\hline \multicolumn{8}{|l|}{ Labour force status } \\
\hline Full time employed & 0.381 & 0.486 & 0.684 & 0.465 & 0.351 & 0.477 & $0.333^{\dagger}$ \\
\hline Part time employed & 0.066 & 0.249 & 0.077 & 0.267 & 0.065 & 0.247 & 0.012 \\
\hline Self employed & 0.375 & 0.484 & 0.063 & 0.243 & 0.406 & 0.491 & $-0.343^{\dagger}$ \\
\hline Not in labour force & 0.178 & 0.382 & 0.176 & 0.381 & 0.178 & 0.382 & -0.001 \\
\hline \multicolumn{8}{|l|}{ Relationship to head } \\
\hline Child & 0.540 & 0.498 & 0.602 & 0.490 & 0.534 & 0.499 & $0.068^{\dagger}$ \\
\hline Partner & 0.079 & 0.269 & 0.118 & 0.323 & 0.075 & 0.263 & $0.043^{\dagger}$ \\
\hline Sibling & 0.245 & 0.430 & 0.173 & 0.379 & 0.253 & 0.435 & $-0.079^{\dagger}$ \\
\hline Other relative & 0.092 & 0.290 & 0.071 & 0.258 & 0.095 & 0.293 & $-0.023 * *$ \\
\hline \multicolumn{8}{|l|}{ Destination country } \\
\hline High income & 0.218 & 0.413 & 0.426 & 0.495 & 0.198 & 0.398 & $0.228^{\dagger}$ \\
\hline Upper-middle income & 0.024 & 0.153 & 0.039 & 0.195 & 0.022 & 0.148 & $0.017 * * *$ \\
\hline Lower-middle income & 0.541 & 0.498 & 0.341 & 0.474 & 0.561 & 0.496 & $-0.220^{\dagger}$ \\
\hline Low income & 0.217 & 0.412 & 0.194 & 0.396 & 0.219 & 0.414 & -0.025 \\
\hline \multicolumn{8}{|l|}{ Origin country } \\
\hline Burkina Faso & 0.189 & 0.392 & 0.009 & 0.093 & 0.207 & 0.406 & $-0.199^{\dagger}$ \\
\hline Kenya & 0.191 & 0.393 & 0.431 & 0.496 & 0.167 & 0.373 & $0.264^{\dagger}$ \\
\hline Nigeria & 0.271 & 0.445 & 0.191 & 0.393 & 0.279 & 0.449 & $-0.088^{\dagger}$ \\
\hline Senegal & 0.222 & 0.416 & 0.120 & 0.325 & 0.233 & 0.422 & $-0.113^{\dagger}$ \\
\hline Uganda & 0.126 & 0.332 & 0.249 & 0.433 & 0.113 & 0.317 & $0.136^{\dagger}$ \\
\hline \multicolumn{8}{|l|}{ Origin $\mathrm{HH}$ characteristics ${ }^{\mathrm{a}}$} \\
\hline Non-remittance income (in 1,000 USD) & 5.239 & 15.122 & 12.917 & 29.982 & 4.470 & 12.457 & $8.447^{\dagger}$ \\
\hline Urban & 0.400 & 0.490 & 0.679 & 0.467 & 0.372 & 0.483 & $0.307^{\dagger}$ \\
\hline Number of migrants & 3.133 & 2.681 & 2.882 & 2.370 & 3.158 & 2.709 & $-0.276^{* *}$ \\
\hline HH head=male & 0.745 & 0.436 & 0.695 & 0.461 & 0.750 & 0.433 & $-0.055 * * *$ \\
\hline House is owned & 0.835 & 0.371 & 0.743 & 0.437 & 0.845 & 0.362 & $-0.101^{\dagger}$ \\
\hline Land is owned & 0.706 & 0.456 & 0.548 & 0.498 & 0.721 & 0.448 & $-0.173^{\dagger}$ \\
\hline Electricity & 0.557 & 0.497 & 0.815 & 0.389 & 0.531 & 0.499 & $0.283^{\dagger}$ \\
\hline HH size & 7.716 & 5.545 & 5.144 & 3.801 & 7.973 & 5.626 & $-2.829^{\dagger}$ \\
\hline Share of dependents & 0.539 & 0.283 & 0.488 & 0.322 & 0.544 & 0.278 & $-0.056^{\dagger}$ \\
\hline Observations & \multicolumn{2}{|c|}{7,536} & \multicolumn{2}{|c|}{686} & \multicolumn{2}{|c|}{6,850} & \\
\hline
\end{tabular}

Notes: ${ }^{a}$ The descriptive statistics for the characteristics of the origin household are calculated at the migrant level. The total amount of remittances is calculated based on all migrants, irrespective of whether they send remittances or not. The last column shows the difference in mean values between migrants with a university degree and migrants without a university degree. Significance stars indicate the result of the respective t-test. ${ }^{\dagger} \mathrm{p}<0.001 ; * * * \mathrm{p}<0.01 ;{ }^{* *} \mathrm{p}<0.05 ;{ }^{*} \mathrm{p}<0.1$. 